\title{
The use of mono- and combination drug therapy in men and women with lower urinary tract symptoms (LUTS) in the UK: a retrospective observational study
}

\author{
Mahmood Ali $^{1,2^{*}{ }^{+}}$, Margarita Landeira ${ }^{2 \dagger}$, Patrick J. O. Covernton ${ }^{2}$, Nurul Choudhury ${ }^{2 \dagger}$, Ashley Jaggi ${ }^{1,2 \dagger}$, \\ Francis Fatoye ${ }^{1}$ and Rob van Maanen ${ }^{3+}$
}

\begin{abstract}
Background: Combination drug therapy for lower urinary tract symptoms (LUTS) is beneficial to selected patients and recommended by guidelines. Patterns of real-world LUTS drug use, especially combination drug therapy, have not been studied extensively. Moreover, further understanding of the recent landscape is required following the introduction of the beta-3-adrenoceptor agonist mirabegron in the UK in 2013 for overactive bladder (OAB). The objective was to describe mono- and combination drug therapy use for LUTS in patients in UK clinical practice.
\end{abstract}

Methods: This was a retrospective, descriptive, observational database study using UK Clinical Practice Research Datalink GOLD and linked databases. Men and women $\geq 18$ years with a first prescription for any LUTS drug from 2014 to 2016 with $\geq 12$ months continuous enrollment pre- and post-index date were included. Primary endpoints were mono- or combination drug therapy use for LUTS in male and female cohorts. Secondary endpoints were description of treatment prescribed, treatment persistence and patient demographics. Data were analyzed descriptively. Subcohorts were defined by drugs prescribed at index date.

Results: 79,472 patients (61.3\% male) were included, based on index treatments. Of all men, $82.5 \%$ received any benign prostatic obstruction (BPO) drug, $25.4 \%$ any $\mathrm{OAB}$ drug, and $7.9 \%$ any BPO drug plus any OAB drug. As either mono- or combination drug therapy, $77.1 \%$ received an al pha-blocker, $18.9 \%$ a 5 -alpha reductase inhibitor, $23.9 \%$ an antimuscarinic agent, and $2.1 \%$ mirabegron. Of all women, $94.5 \%$ received any OAB drug, $6.0 \%$ duloxetine, and $0.5 \%$ any $\mathrm{OAB}$ drug plus duloxetine. As either mono- or combination drug therapy, $87.7 \%$ received an antimuscarinic, and $9.7 \%$ mirabegron. In men or women receiving $O A B$ treatment, approximately $2.5 \%$ received combination drug therapy with an antimuscarinic agent and mirabegron. For $O A B$ drug monotherapies, mirabegron had the highest persistence in both male and female cohorts.

Conclusions: This study provides a better understanding of the recent landscape of LUTS drug use in UK clinical practice. It highlights potential undertreatment of storage symptoms in men with LUTS and the low use of combination $\mathrm{OAB}$ treatments.

\footnotetext{
*Correspondence: mmd.ali01@gmail.com

${ }^{\dagger}$ At the time of study or manuscript preparation

${ }^{1}$ Manchester Metropolitan University, Manchester, UK

Full list of author information is available at the end of the article
} original author(s) and the source, provide a link to the Creative Commons licence, and indicate if changes were made. The images or other third party material in this article are included in the article's Creative Commons licence, unless indicated otherwise in a credit line to the material. If material is not included in the article's Creative Commons licence and your intended use is not permitted by statutory regulation or exceeds the permitted use, you will need to obtain permission directly from the copyright holder. To view a copy of this licence, visit http://creativecommons.org/licenses/by/4.0/. The Creative Commons Public Domain Dedication waiver (http://creativeco mmons.org/publicdomain/zero/1.0/) applies to the data made available in this article, unless otherwise stated in a credit line to the data. 
Keywords: Benign prostatic obstruction (BPO), Overactive bladder (OAB), Lower urinary tract symptoms (LUTS), Persistence, Stress urinary incontinence (SUI)

\section{Background}

Lower urinary tract symptoms (LUTS) is an overarching term for symptoms in men and women, comprising storage, voiding and post-voiding components [1,2]. In both men and women, storage LUTS are commonly attributed to overactive bladder $(\mathrm{OAB})$ syndrome [1-3], which is defined as urinary urgency, usually with increased daytime frequency and/or nocturia, with/without urinary incontinence, and with no urinary tract infection or other detectable disease $[1,2,4]$. Stress urinary incontinence (SUI) is another common cause of LUTS, especially in women, and involves involuntary urine leakage associated with physical activity (e.g., coughing, sneezing), often as a consequence of childbirth $[1,3,5]$. Voiding LUTS in men are commonly attributed to benign prostatic obstruction (BPO: bladder outlet obstruction [BOO] due to benign prostatic enlargement) $[1,6]$.

Although conservative treatment, including lifestyle intervention and behavioral therapies (such as bladder training), remains the foundation of LUTS management, several pharmacological treatments are available [6-8]. If conservative treatment fails, pharmacological therapy for $\mathrm{OAB} /$ urgency urinary incontinence (UUI) includes antimuscarinic agents or the beta-3 agonist, mirabegron [7]. Combination of an antimuscarinic agent plus mirabegron has also been shown to be effective [9] and is recommended as an option if patients respond inadequately to monotherapy $[7,8]$. In patients with SUI where surgery is not indicated, duloxetine is the only recommended pharmacotherapy [7]; it is unknown to what extent duloxetine is used in combination with $\mathrm{OAB}$ drugs. Women with LUTS can experience symptoms of both OAB and SUI [10], but there are no recommendations regarding combination therapy with $\mathrm{OAB} / \mathrm{UUI}$ drugs for such patients.

In men with LUTS suggestive of BPO, the main treatment options include alpha-blockers for rapid symptomatic relief $[11,12]$ and 5-alpha reductase inhibitors (5-ARIs) to delay progression of BPO and help manage symptoms over the long term in men at risk of disease progression $[6,12]$. Alpha-blockers are usually used as first-line treatment [6], but the European Association of Urology (EAU) guidelines recommend combined alphablocker and 5-ARI treatment in men with moderate-tosevere LUTS and an increased risk of disease progression [6]. However, many men with LUTS (approximately 50\%) experience mixed symptoms suggestive of both $\mathrm{OAB}$ and BPO [13]. Only one-third of these men with mixed symptoms will achieve adequate symptom control with an alpha-blocker alone, with the remainder requiring additional pharmacotherapy to manage residual storage LUTS [14]. Clinical trials in men on alpha-blocker monotherapy who still have bothersome storage LUTS have shown that adding an $\mathrm{OAB}$ drug can significantly reduce storage symptoms and improve quality of life [15-18]. The addition of an antimuscarinic agent or beta-3-agonist if storage symptoms are not relieved by alpha-blocker monotherapy is a recommended treatment strategy in clinical guidelines [6].

Patterns of real-world LUTS drug use, especially combination drug therapy, have not been studied extensively in the UK. Our study investigated the recent landscape of pharmacotherapy for men and women with LUTS in UK clinical practice, including the types and extent of combination therapies used, and persistence with treatment.

\section{Methods}

\section{Study design}

This was a retrospective, descriptive, observational database study of LUTS treatment in the UK primary care setting. Data were extracted from the UK Clinical Practice Research Datalink (CPRD) GOLD, a national longitudinal primary care database, which contains anonymized electronic health records of over 15.6 million patients (September 2018 version) [19]. Only de-identified data were obtained, and patients could opt out if they did not wish to have their data used for research purposes. CPRD GOLD was linked to the Hospital Episode Statistic database in England for the exploration of resource use, and the Index of Multiple Deprivation (IMD). The IMD provides an indication of patients' socio-economic status measured at the GP surgery level. All methods were carried out in accordance with relevant guidelines and regulations.

\section{Population}

The study included adults ( $\geq 18$ years of age) identified with LUTS (evidenced by prescription of drugs used to treat LUTS) between 1 January 2014 and 31 December 2016 , with $\geq 12$ months continuous enrollment preand post-index date (index date was the date on which patients were prescribed a new index pharmacotherapy [one pharmacotherapy or combination of two or more pharmacotherapies] for the first time between 1 January 2014 and 31 December 2016). Patients prescribed the same index drug in the 12-month pre-index period were excluded. Male and female cohorts were considered 
separately. Sub-cohorts were defined by the drugs received at index date (Additional file 1: Table S1), and combination therapy was categorized by index drug with patients placed into one sub-cohort only. If $>1$ drug was prescribed at index date and this indicated assignment to different sub-cohorts, the order of preference was as follows: LUTS (including $\mathrm{OAB}$ ) had priority over BPO in the male cohort and LUTS (including OAB) over SUI in the female cohort. Patients were classified as being on combination therapy if the additional drug(s) was prescribed within the prescription duration of the first drug (prescription duration was calculated by total tablets prescribed divided by total daily dose) and the index date was the first prescription of the most recently prescribed drug.

The LUTS (including OAB) cohort were patients receiving an $\mathrm{OAB}$ drug (antimuscarinic agent and/or mirabegron), with or without a BPO drug (alpha-blocker and/or 5-ARI) in the male cohort and with or without a SUI drug (duloxetine) in the female cohort. BPO patients were those receiving an alpha-blocker and/or 5-ARI without an $\mathrm{OAB}$ drug. SUI patients were those receiving duloxetine without an $\mathrm{OAB}$ drug (Additional file 1: Table S1).

\section{Endpoints}

Primary endpoints were use of mono- or combination drug therapy for LUTS in a) male or b) female cohorts. Secondary endpoints were socio-demographic and clinical characteristics, and description of monotherapy/combination drugs. Exploratory variables included treatment persistence and presence of a LUTS diagnostic Read code in the pre-index period.

\section{Data analyses}

Socio-demographic characteristics were recorded by age, sex and IMD; clinical characteristics were recorded by comorbidity (number of chronic diseases from the Quality and Outcomes Framework [QOF]), polypharmacy (number of distinct British National Formulary headers from CPRD GOLD), and antimuscarinic treatment experience ( $\geq 1$ antimuscarinic agent prescription, other than index treatment, in CPRD GOLD), within the 12-month pre-index period.

\section{Statistical analyses}

Data were analyzed descriptively for the overall population and by cohort and sub-cohort. Analyses were conducted using SAS Studio version 3.5.

Persistence was analyzed by median time (from index date) to first discontinuation (TTD, during the 12-month post-index period), and persistence rate at 12 months, calculated using the Kaplan-Meier method.
Discontinuation was defined as exceeding the maximum allowable gap duration (MAGD) between prescriptions. For the base-case, the MAGD was defined as 1.5 times the estimated duration of the most recent prescription. Combination treatment was classed as discontinued upon discontinuation of any one of the component drugs. Data were classed as Not Observable when the number of patients still at risk was below the $20 \%$ of the initial sample threshold required to allow for persistence to be calculated or, the median was not reached.

\section{Sensitivity analyses}

Sensitivity analyses (SA1-7) varied the combination drug therapy definition, MAGD definition and presence of LUTS diagnostic code. The combination drug therapy definition was changed to: a second drug of interest is prescribed within 1.5 times the estimated prescription interval of the first drug (SA1); two drugs of interest are prescribed on the same day (SA2); both drugs continue to be prescribed for $\geq 90$ days from index date (SA3). MAGD definition was changed to: equivalent to (SA4) or double (SA5) the length of most recent prescription. To be considered a patient with LUTS, patients required a LUTS diagnosis code (Additional file 1: Tables S2a-c) registered any time before the index date (SA6). In the final sensitivity analysis, patients diagnosed with hypertension were excluded (SA7).

\section{Results \\ Patients}

Of 223,831 patients with one or more LUTS drug prescription between 1 January 2014 and 31 December 2016, 79,472 were included in the study $(61.3 \%[n=48,690]$ in the male and $38.7 \%$ [ $n=30,782]$ in the female cohort) (Fig. 1) (Additional file 1: Table S3a and b).

At index date, the mean age was 65.5 years. In the 12-month pre-index period, patients had a mean of 0.2 new diagnoses of QOF chronic diseases and were prescribed a mean of 9.6 drugs; $5.1 \%$ of patients had received an antimuscarinic agent (Tables 1,2). Approximately one in five patients $(16.3 \%)$ had a LUTS diagnostic code, the notable exceptions being for doxazosin and duloxetine monotherapy (3\% each).

\section{Extent of monotherapy and combination drug use}

Of all men $(n=48,690), 82.5 \%$ received any BPO drug (alpha-blocker and/or 5-ARI), 74.6\% received any (one or more) BPO drug without an OAB drug, 25.4\% received any $\mathrm{OAB}$ drug (antimuscarinic agent and/or mirabegron) (17.5\% received any [one or more] OAB drug without a $\mathrm{BPO}$ drug, and $7.9 \%$ received any $\mathrm{BPO}$ drug plus any $\mathrm{OAB}$ drug). As either mono- or combination therapy, $77.1 \%$ were receiving an alpha-blocker, $18.9 \%$ received a 


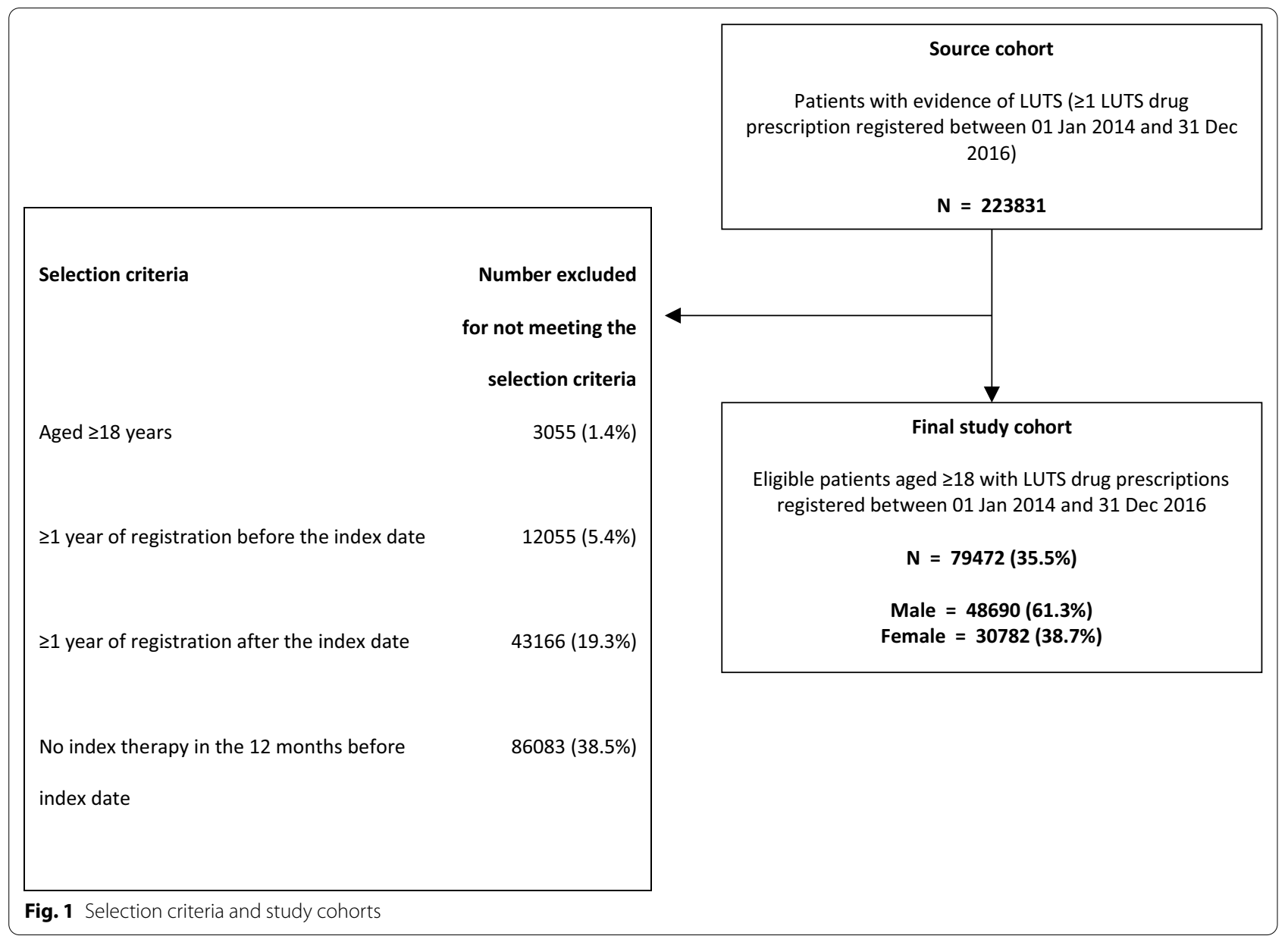

5-ARI, $23.9 \%$ an antimuscarinic agent and $2.1 \%$ mirabegron. The most common drug received either as monoor combination therapy was tamsulosin $(61.2 \%$ of all men) and the most common combination was finasteride plus tamsulosin (8.6\%, with or without additional drugs). Of all women $(n=30,782), 94.5 \%$ received any OAB drug, $6.0 \%$ received a SUI drug, and $0.5 \%$ received any $\mathrm{OAB}$ drug plus a SUI drug (Additional file 1: Table S3b). As either mono- or combination therapy, $87.7 \%$ were receiving an antimuscarinic agent and $9.7 \%$ mirabegron. The extent of drug class use is summarized in Tables 3, 4 and 5, and in Additional file 1: Tables S3b and 4.

\section{Male LUTS (including OAB) sub-cohort}

In this sub-cohort of 12,383 men treated with any $O A B$ drug, $64.2 \%$ received monotherapy with an $\mathrm{OAB}$ drug and $35.8 \%$ received combinations $(31.2 \%$ with any $\mathrm{OAB}$ drug plus any BPO drug and $7.8 \%$ with two or more OAB drugs [3.2\% with and $4.6 \%$ without a BPO drug]) (Table 3). In addition, $94.1 \%$ received any antimuscarinic agent, $8.4 \%$ received mirabegron, $2.5 \%$ received any antimuscarinic agent plus mirabegron and 5.4\% received two or more antimuscarinic agents (Table 3). In this sub-cohort $26.9 \%$ of patients received combination therapy including any alpha-blocker and any antimuscarinic agent and $2.6 \%$ received any alpha-blocker plus mirabegron. Furthermore, $7.7 \%$ of this sub-cohort received triple therapy with any alpha-blocker plus any 5-ARI plus any OAB drug (Table 3).

The most frequently prescribed $\mathrm{OAB}$ monotherapies in this sub-cohort were solifenacin $(22.3 \%)$, oxybutynin (21.1\%), tolterodine (11.7\%) and mirabegron (4.1\%) (Table 3; Additional file 1: Table S5).

The most common $\mathrm{OAB}$ drug either alone or in combination was solifenacin $(40.6 \%$ of the OAB subcohort). The most frequently prescribed combination was solifenacin plus tamsulosin $(7.3 \%$ of sub-cohort for this two-drug combination alone; $11.7 \%$ when also including additional drugs), followed by oxybutynin plus tamsulosin and then tolterodine plus tamsulosin, with $2.9 \%$ and $2.0 \%$ of sub-cohort, respectively. A total of $0.8 \%$ of this sub-cohort received mirabegron plus solifenacin alone $(1.4 \%$ when also including additional drugs). 
Table 1 Population demographic and clinical characteristics for male sub-cohorts

\begin{tabular}{|c|c|c|c|}
\hline & & $\begin{array}{l}\text { LUTS (including } O A B \text { ) } \\
\mathrm{N}=12,383\end{array}$ & $\begin{array}{l}\text { BPO only } \\
\mathrm{N}=36,307\end{array}$ \\
\hline \multirow[t]{9}{*}{ Age at index date } & $\mathrm{n}$ & 12,383 & 36,307 \\
\hline & Mean (SD) & $65.54(15.73)$ & $67.98(12.47)$ \\
\hline & $18-24$ & $192(1.6 \%)$ & $119(0.3 \%)$ \\
\hline & $25-34$ & $434(3.5 \%)$ & $356(1.0 \%)$ \\
\hline & $35-44$ & $736(5.9 \%)$ & $997(2.7 \%)$ \\
\hline & $45-54$ & $1438(11.6 \%)$ & $3586(9.9 \%)$ \\
\hline & $55-64$ & $2188(17.7 \%)$ & $7746(21.3 \%)$ \\
\hline & $65-74$ & $3379(27.3 \%)$ & $11,905(32.8 \%)$ \\
\hline & $\geq 75$ & $4016(32.4 \%)$ & $11,598(31.9 \%)$ \\
\hline \multirow[t]{6}{*}{ Index of multiple deprivation at index prescription (GP surgery level) } & $\mathrm{n}$ & 5112 & 16,534 \\
\hline & $1=$ least deprived & $633(12.4 \%)$ & $2770(16.8 \%)$ \\
\hline & 2 & $1124(22.0 \%)$ & $3654(22.1 \%)$ \\
\hline & 3 & $968(18.9 \%)$ & $3175(19.2 \%)$ \\
\hline & 4 & $963(18.8 \%)$ & $2926(17.7 \%)$ \\
\hline & $5=$ most deprived & 1424 (27.9\%) & 4009 (24.2\%) \\
\hline New comorbidities & $\mathrm{n}$ & 12,383 & 36,307 \\
\hline \multirow{5}{*}{$\begin{array}{l}\text { Count of newly diagnosed chronic diseases from the QOF within the } \\
12 \text {-month pre-index period }\end{array}$} & Mean (SD) & $0.21(0.50)$ & $0.22(0.51)$ \\
\hline & 0 & $10,228(82.6 \%)$ & $29,480(81.2 \%)$ \\
\hline & 1 & 1790 (14.5\%) & $5700(15.7 \%)$ \\
\hline & 2 & $313(2.5 \%)$ & $978(2.7 \%)$ \\
\hline & $3+$ & $52(0.4 \%)$ & $149(0.4 \%)$ \\
\hline Polypharmacy & $\mathrm{n}$ & 12,383 & 36,307 \\
\hline \multirow[t]{6}{*}{ Number of distinct BNF headers within the 12-month pre-index period } & Mean (SD) & $9.81(7.27)$ & $8.52(6.45)$ \\
\hline & 0 & $576(4.7 \%)$ & $1757(4.8 \%)$ \\
\hline & {$[1 ; 3]$} & $1931(15.6 \%)$ & $6668(18.4 \%)$ \\
\hline & {$[4 ; 7]$} & $2936(23.7 \%)$ & $10,342(28.5 \%)$ \\
\hline & {$[8 ; 19]$} & $5707(46.1 \%)$ & $15,220(41.9 \%)$ \\
\hline & $20+$ & $1233(10.0 \%)$ & $2320(6.4 \%)$ \\
\hline \multirow[t]{3}{*}{ Antimuscarinic treatment experience within the 12-month pre-index period } & $\mathrm{n}$ & 12,383 & 36,307 \\
\hline & Yes & $908(7.3 \%)$ & $397(1.1 \%)$ \\
\hline & No & $11,475(92.7 \%)$ & $35,910(98.9 \%)$ \\
\hline
\end{tabular}

Male LUTS (including OAB) sub-cohort patients were those receiving an OAB drug (antimuscarinic and/or mirabegron) with or without a BPO drug (either an alphablocker and/or 5-ARI). Male BPO sub-cohort patients were those receiving an alpha-blocker and/or 5-ARI without an OAB drug. Percentages may not total exactly 100 due to rounding

BNF: British National Formulary; BPO: benign prostatic obstruction; GP: general practitioner; LUTS: lower urinary tract symptoms; OAB: overactive bladder; QOF: Quality and Outcomes Framework; SD: standard deviation

\section{Male BPO sub-cohort}

In this sub-cohort of 36,307 men, $81.9 \%$ received monotherapy $(75.6 \%$ with any alpha-blocker; $6.3 \%$ with any 5 -ARI) and $18.1 \%$ received combination therapy (15.3\% with any alpha-blocker plus any 5-ARI). Tamsulosin monotherapy was the most commonly prescribed (58.3\% of sub-cohort patients), followed by doxazosin (15.0\%) (Table 4; Additional file 1: Table S6). For combination therapy, $49.7 \%$ of combination therapy patients were prescribed finasteride plus tamsulosin $(9.0 \%$ of this sub-cohort for these two drugs alone; $10.0 \%$ when also including additional drugs), followed by dutasteride plus tamsulosin and doxazosin plus tamsulosin. In addition, 93.6\% of patients in this sub-cohort overall received an alpha-blocker and $21.7 \%$ a 5 -ARI.

\section{Female LUTS (including OAB) sub-cohort}

In this sub-cohort of 29,094 women, $90.5 \%$ of patients received monotherapy (83.4\% any antimuscarinic, $7.1 \%$ mirabegron) and $9.5 \%$ combination therapy (9.0\% with two or more $\mathrm{OAB}$ drugs). In addition, $0.5 \%$ of women in this sub-cohort received any $\mathrm{OAB}$ drug plus duloxetine 
Table 2 Population demographic and clinical characteristics for female sub-cohorts

\begin{tabular}{|c|c|c|c|}
\hline & & $\begin{array}{l}\text { LUTS (including OAB) } \\
\mathrm{N}=29,094\end{array}$ & SUI N $=1688$ \\
\hline \multirow[t]{9}{*}{ Age at index date } & $\mathrm{n}$ & 29,094 & 1688 \\
\hline & Mean (SD) & $62.92(16.77)$ & $54.24(16.29)$ \\
\hline & $18-24$ & $604(2.1 \%)$ & $32(1.9 \%)$ \\
\hline & $25-34$ & $1121(3.9 \%)$ & $159(9.4 \%)$ \\
\hline & $35-44$ & $2536(8.7 \%)$ & $315(18.7 \%)$ \\
\hline & $45-54$ & $4835(16.6 \%)$ & $399(23.6 \%)$ \\
\hline & $55-64$ & $5264(18.1 \%)$ & $315(18.7 \%)$ \\
\hline & $65-74$ & $6446(22.2 \%)$ & $248(14.7 \%)$ \\
\hline & $\geq 75$ & $8288(28.5 \%)$ & $220(13.0 \%)$ \\
\hline \multirow[t]{6}{*}{ Index of multiple deprivation at index prescription (GP surgery level) } & $\mathrm{n}$ & 11,999 & 665 \\
\hline & $1=$ least deprived & $1534(12.8 \%)$ & $86(12.9 \%)$ \\
\hline & 2 & $2418(20.2 \%)$ & $148(22.3 \%)$ \\
\hline & 3 & $2292(19.1 \%)$ & $137(20.6 \%)$ \\
\hline & 4 & $2285(19.0 \%)$ & $105(15.8 \%)$ \\
\hline & $5=$ most deprived & $3470(28.9 \%)$ & $189(28.4 \%)$ \\
\hline New comorbidities & n & 29,094 & 1688 \\
\hline \multirow{5}{*}{$\begin{array}{l}\text { Count of newly diagnosed chronic diseases from the QOF within the 12-month } \\
\text { pre-index period }\end{array}$} & Mean (SD) & $0.20(0.48)$ & $0.23(0.53)$ \\
\hline & 0 & $24,327(83.6 \%)$ & $1374(81.4 \%)$ \\
\hline & 1 & $3950(13.6 \%)$ & $248(14.7 \%)$ \\
\hline & 2 & $712(2.4 \%)$ & $56(3.3 \%)$ \\
\hline & $3+$ & $105(0.4 \%)$ & $10(0.6 \%)$ \\
\hline Polypharmacy & $\mathrm{n}$ & 29,094 & 1688 \\
\hline \multirow[t]{6}{*}{ Number of distinct BNF headers within the 12-month pre-index period } & Mean (SD) & $10.77(7.49)$ & $11.55(7.42)$ \\
\hline & 0 & $681(2.3 \%)$ & $29(1.7 \%)$ \\
\hline & {$[1 ; 3]$} & $3766(12.9 \%)$ & $165(9.8 \%)$ \\
\hline & {$[4 ; 7]$} & $6944(23.9 \%)$ & $384(22.7 \%)$ \\
\hline & {$[8 ; 19]$} & $14,178(48.7 \%)$ & $873(51.7 \%)$ \\
\hline & $20+$ & $3525(12.1 \%)$ & $237(14.0 \%)$ \\
\hline \multirow[t]{3}{*}{ Antimuscarinic treatment experience within the 12-month pre-index period } & $\mathrm{n}$ & 29,094 & 1688 \\
\hline & Yes & $2681(9.2 \%)$ & $56(3.3 \%)$ \\
\hline & No & $26,413(90.8 \%)$ & $1632(96.7 \%)$ \\
\hline
\end{tabular}

Female LUTS (including OAB) sub-cohort patients were those receiving an OAB drug (antimuscarinic and/or mirabegron) with or without a SUI drug (duloxetine). Female SUI sub-cohort patients were those receiving duloxetine without an OAB drug. Percentages may not total exactly 100 due to rounding

BNF: British National Formulary; GP: general practitioner; LUTS: lower urinary tract symptoms; OAB: overactive bladder; QOF: Quality and Outcomes Framework; SD: standard deviation; SUI: stress urinary incontinence

(Table 5). Mirabegron plus duloxetine was prescribed to $0.04 \%$ of patients in this sub-cohort. A combination of any two or more antimuscarinic agents ( \pm mirabegron) was used by $6.3 \%$ of sub-cohort patients and any antimuscarinic agent plus mirabegron by $2.7 \%$. The most common $\mathrm{OAB}$ drug either alone or in combination was solifenacin $(41.0 \%$ of the OAB sub-cohort) and $10.2 \%$ of this sub-cohort overall received mirabegron.

Solifenacin was the most frequently prescribed monotherapy (34.7\% of this sub-cohort), followed by oxybutynin and tolterodine (Table 5; Additional file 1: Table S7); $92.8 \%$ of sub-cohort patients were prescribed any antimuscarinic agent. The most frequently prescribed combinations were solifenacin plus tolterodine, oxybutynin plus solifenacin and mirabegron plus solifenacin.

\section{Female SUI sub-cohort}

Duloxetine was prescribed to all $(n=1688)$ sub-cohort patients and was the only drug used in women with SUI, as defined in the base-case.

\section{Persistence}

Kaplan Meier curves of TTD by mono- and combination drug therapy for the male BPO sub-cohort, and for 
Table 3 Extent of mono- and combination drug therapy use in the male LUTS (including OAB) sub-cohort

\begin{tabular}{|c|c|c|c|c|c|c|}
\hline \multirow{2}{*}{$\begin{array}{l}\text { Monotherapy } \\
\text { Any OAB drug monotherapy }\end{array}$} & \multirow{2}{*}{$\begin{array}{l}\mathbf{N} \\
7946\end{array}$} & \multicolumn{2}{|c|}{$\begin{array}{l}\% \text { of monotherapy } \\
\text { patients }\end{array}$} & \multicolumn{2}{|c|}{$\begin{array}{l}\% \text { of all patients in male } O A B \\
\text { sub-cohort }\end{array}$} & \multirow{2}{*}{$\begin{array}{l}\begin{array}{l}\% \text { of all } \\
\text { male } \\
\text { patients }\end{array} \\
16.3\end{array}$} \\
\hline & & 100.0 & & \multicolumn{2}{|l|}{64.2} & \\
\hline Any antimuscarinic monotherapy & 7443 & \multicolumn{2}{|l|}{93.7} & \multicolumn{2}{|l|}{60.1} & 15.3 \\
\hline Solifenacin & 2759 & \multicolumn{2}{|l|}{34.7} & \multicolumn{2}{|l|}{22.3} & 5.7 \\
\hline Oxybutynin & 2613 & \multicolumn{2}{|l|}{32.9} & \multicolumn{2}{|l|}{21.1} & 5.4 \\
\hline Tolterodine & 1445 & \multicolumn{2}{|l|}{18.2} & \multicolumn{2}{|l|}{11.7} & 3.0 \\
\hline Mirabegron & 503 & \multicolumn{2}{|l|}{6.3} & \multicolumn{2}{|l|}{4.1} & 1.0 \\
\hline Fesoterodine & 285 & \multicolumn{2}{|l|}{3.6} & 2.3 & & 0.6 \\
\hline Trospium & 227 & 2.9 & & 1.8 & & 0.5 \\
\hline Flavoxate & 49 & 0.6 & & 0.4 & & 0.1 \\
\hline Darifenacin & 47 & 0.6 & & 0.4 & & 0.1 \\
\hline Propiverine & 18 & 0.2 & & 0.1 & & 0.04 \\
\hline Combination drug therapy & & $\mathrm{N}$ & $\begin{array}{l}\% \text { of co } \\
\text { therapy }\end{array}$ & $\begin{array}{l}\text { tion drug } \\
\text { nts }\end{array}$ & $\begin{array}{l}\% \text { of all patients in } \\
\text { male } O A B \text { sub-cohort }\end{array}$ & $\begin{array}{l}\% \text { of all } \\
\text { male } \\
\text { patients }\end{array}$ \\
\hline Any combination drug therapy & & 4437 & 100.0 & & 35.8 & 9.1 \\
\hline Any BPO drug + any OAB drug & & 3863 & 87.1 & & 31.2 & 7.9 \\
\hline Any BPO drug + any antimuscarinic & & 3643 & 82.1 & & 29.4 & 7.5 \\
\hline Any BPO drug + mirabegron & & 347 & 7.8 & & 2.8 & 0.7 \\
\hline Any alpha-blocker + any OAB drug & & 3538 & 79.7 & & 28.6 & 7.3 \\
\hline Any alpha-blocker + any antimusca & begron and/or 5-ARI) & 3330 & 75.1 & & 26.9 & 6.8 \\
\hline Any alpha-blocker + mirabegron $( \pm$ & nic and/or 5-ARI) & 326 & 7.3 & & 2.6 & 0.7 \\
\hline Triple drug therapy (any alpha-block & $A R I+$ any $O A B$ drug) & 958 & 21.6 & & 7.7 & 2.0 \\
\hline Any OAB drug + any 5-ARI ( \pm alpha- & & 1284 & 28.9 & & 10.4 & 2.6 \\
\hline Any OAB drug + any 5-ARI (excl. alp & & 325 & 7.3 & & 2.6 & 0.7 \\
\hline Mirabegron + any antimuscarinic $( \pm$ & & 307 & 6.9 & & 2.5 & 0.6 \\
\hline Any $\geq 2 \mathrm{OAB}( \pm \mathrm{BPO}$ drug $)$ & & 964 & 21.7 & & 7.8 & 2.0 \\
\hline Any $\geq 2 \mathrm{OAB}$ (no BPO drug) & & 574 & 12.9 & & 4.6 & 1.2 \\
\hline Any $\geq 2$ antimuscarinics $( \pm \mathrm{BPO}$ dru & rabegron) & 666 & 15.0 & & 5.4 & 1.4 \\
\hline Solifenacin + tamsulosin & & 902 & 20.3 & & 7.3 & 1.9 \\
\hline Oxybutynin + tamsulosin & & 364 & 8.2 & & 2.9 & 0.7 \\
\hline Tamsulosin + tolterodine & & 248 & 5.6 & & 2.0 & 0.5 \\
\hline Finasteride + solifenacin + tamsulos & & 233 & 5.3 & & 1.9 & 0.5 \\
\hline Doxazosin + solifenacin & & 114 & 2.6 & & 0.9 & 0.2 \\
\hline Solifenacin + tolterodine & & 114 & 2.6 & & 0.9 & 0.2 \\
\hline Finasteride + oxybutynin + tamsulo & & 111 & 2.5 & & 0.9 & 0.2 \\
\hline Oxybutynin + solifenacin & & 102 & 2.3 & & 0.8 & 0.2 \\
\hline Finasteride + solifenacin & & 96 & 2.2 & & 0.8 & 0.2 \\
\hline Mirabegron + tamsulosin & & 96 & 2.2 & & 0.8 & 0.2 \\
\hline Doxazosin + oxybutynin & & 95 & 2.1 & & 0.8 & 0.2 \\
\hline Mirabegron + solifenacin & & 95 & 2.1 & & 0.8 & 0.2 \\
\hline Fesoterodine + tamsulosin & & 83 & 1.9 & & 0.7 & 0.2 \\
\hline Dutasteride + solifenacin + tamsulo & & 79 & 1.8 & & 0.6 & 0.2 \\
\hline Doxazosin + tolterodine & & 69 & 1.6 & & 0.6 & 0.1 \\
\hline Other combinations & & 1636 & 36.9 & & 13.2 & 3.4 \\
\hline Drug class & $\mathrm{N}$ & & & atients in & male OAB sub-cohort & $\begin{array}{l}\% \text { of all } \\
\text { male } \\
\text { patients }\end{array}$ \\
\hline Any $O A B$ drug & 12,383 & & 10 & & & 25.4 \\
\hline
\end{tabular}


Table 3 (continued)

\begin{tabular}{lccc}
\hline Drug class & N & \% of all patients in male OAB sub-cohort & $\begin{array}{c}\text { \% of all } \\
\text { male } \\
\text { patients }\end{array}$ \\
\hline Any OAB drug (no BPO drug) & & 6520 & 68.8 \\
Any antimuscarinic & 11,653 & 94.1 & 17.5 \\
Total mirabegron & 1039 & 8.4 & 23.9 \\
Any alpha-blocker & 3538 & 28.6 & 2.1 \\
Any 5-ARl & 1284 & 10.4 & 7.3 \\
\hline
\end{tabular}

5-ARI: 5-alpha reductase inhibitor; $B P O$ : benign prostatic obstruction; LUTS: lower urinary tract symptoms; $O A B$ : overactive bladder

Table 4 Extent of mono- and combination drug therapy use in the male BPO sub-cohort

\begin{tabular}{|c|c|c|c|c|c|}
\hline Monotherapy & $\mathrm{N}$ & \multicolumn{2}{|c|}{$\begin{array}{l}\% \text { of monotherapy } \\
\text { patients }\end{array}$} & $\begin{array}{l}\% \text { of all patients in BPO sub- } \\
\text { cohort }\end{array}$ & $\begin{array}{l}\% \text { of all } \\
\text { male } \\
\text { patients }\end{array}$ \\
\hline Any BPO drug monotherapy & 29,739 & \multicolumn{2}{|l|}{100.0} & 81.9 & 61.1 \\
\hline Any alpha-blocker monotherapy & 27,462 & \multicolumn{2}{|l|}{92.3} & 75.6 & 56.4 \\
\hline Any 5-ARI monotherapy & 2277 & \multicolumn{2}{|l|}{7.7} & 6.3 & 4.7 \\
\hline Tamsulosin & 21,158 & \multicolumn{2}{|l|}{71.1} & 58.3 & 43.5 \\
\hline Doxazosin & 5456 & \multicolumn{2}{|l|}{18.3} & 15.0 & 11.2 \\
\hline Finasteride & 2131 & \multicolumn{2}{|l|}{7.2} & 5.9 & 4.4 \\
\hline Alfuzosin & 665 & \multicolumn{2}{|l|}{2.2} & 1.8 & 1.4 \\
\hline Dutasteride & 146 & \multicolumn{2}{|l|}{0.5} & 0.4 & 0.3 \\
\hline Prazosin & 101 & \multicolumn{2}{|l|}{0.3} & 0.3 & 0.2 \\
\hline Terazosin & 44 & \multicolumn{2}{|l|}{0.1} & 0.1 & 0.1 \\
\hline Indoramin & 38 & \multicolumn{2}{|l|}{0.1} & 0.1 & 0.1 \\
\hline Combination drug therapy & $\mathrm{N}$ & \multicolumn{2}{|c|}{$\begin{array}{l}\% \text { of combination drug } \\
\text { therapy patients }\end{array}$} & $\begin{array}{l}\% \text { of all patients in BPO } \\
\text { sub-cohort }\end{array}$ & $\begin{array}{l}\% \text { of all } \\
\text { male } \\
\text { patients }\end{array}$ \\
\hline Any combination drug therapy & 6568 & \multicolumn{2}{|l|}{100.0} & 18.1 & 13.5 \\
\hline Any alpha-blocker + any 5-ARI & 5573 & \multicolumn{2}{|l|}{84.9} & 15.3 & 11.4 \\
\hline Finasteride + tamsulosin & 3262 & \multicolumn{2}{|l|}{49.7} & 9.0 & 6.7 \\
\hline Dutasteride + tamsulosin & 1189 & \multicolumn{2}{|l|}{18.1} & 3.3 & 2.4 \\
\hline Doxazosin + tamsulosin & 614 & \multicolumn{2}{|l|}{9.3} & 1.7 & 1.3 \\
\hline Doxazosin + finasteride & 289 & \multicolumn{2}{|l|}{4.4} & 0.8 & 0.6 \\
\hline Alfuzosin + finasteride & 233 & \multicolumn{2}{|l|}{3.5} & 0.6 & 0.5 \\
\hline Alfuzosin + tamsulosin & 208 & \multicolumn{2}{|l|}{3.2} & 0.6 & 0.4 \\
\hline Doxazosin + finasteride + tamsulosin & 167 & \multicolumn{2}{|l|}{2.5} & 0.5 & 0.3 \\
\hline Dutasteride + finasteride + tamsulosin & 125 & \multicolumn{2}{|l|}{1.9} & 0.3 & 0.3 \\
\hline Other combinations & 481 & \multicolumn{2}{|l|}{7.3} & 1.3 & 1.0 \\
\hline Drug class & $\mathbf{N}$ & & \multicolumn{2}{|c|}{$\%$ of all patients in BPO sub-cohort } & $\begin{array}{l}\% \text { of all } \\
\text { male } \\
\text { patients }\end{array}$ \\
\hline Any BPO drug & 36,307 & & 100 & & 74.6 \\
\hline Any alpha-blocker & 33,984 & & 93.6 & & 69.8 \\
\hline Any 5-ARI & 7896 & & 21.7 & & 16.2 \\
\hline
\end{tabular}

5-ARI: 5-alpha reductase inhibitor; $B P O$ : benign prostatic obstruction 
Table 5 Extent of mono- and combination drug therapy use in the female LUTS (including OAB) sub-cohort

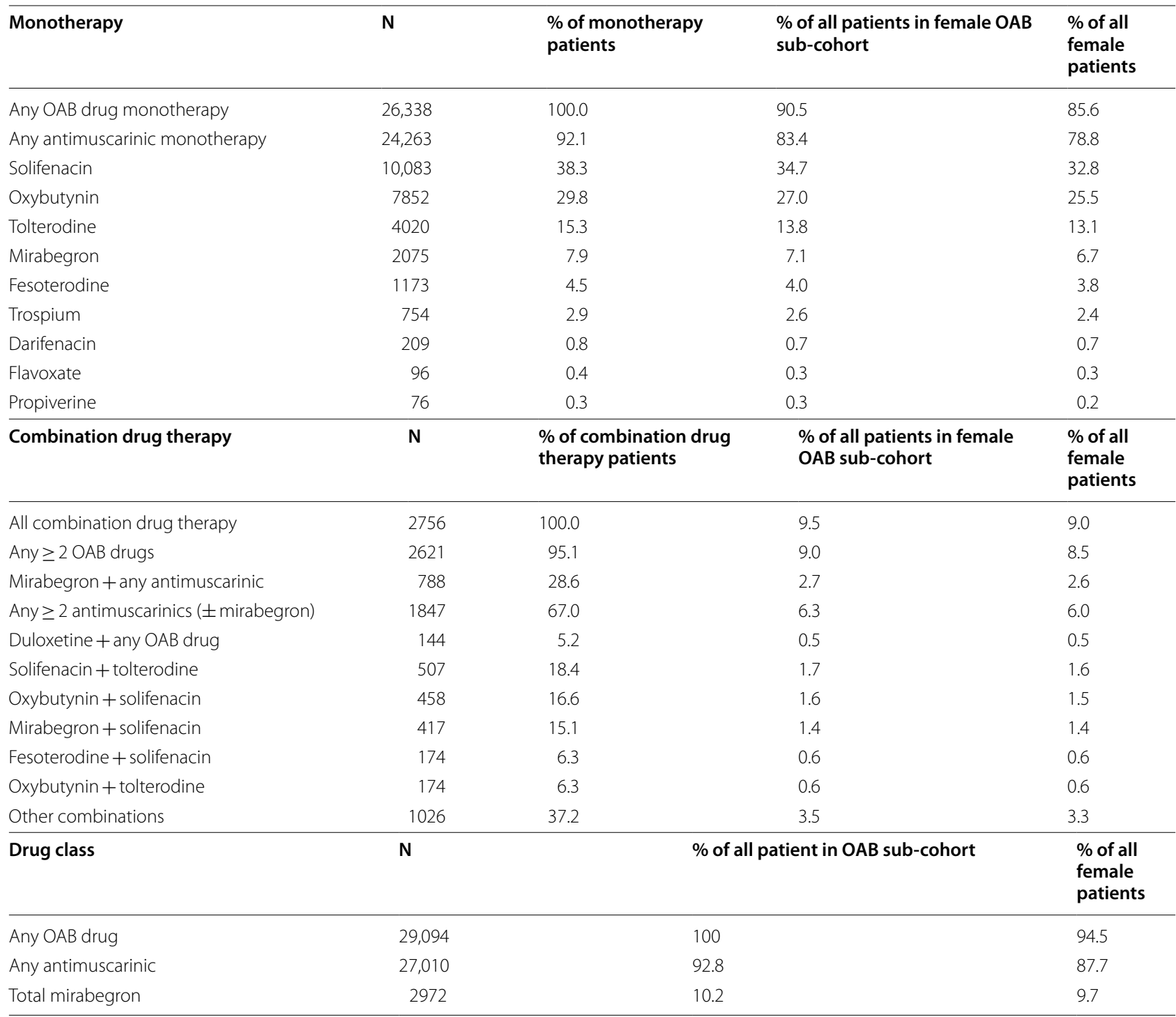

LUTS: lower urinary tract symptoms; $O A B$ : overactive bladder

duloxetine in the female SUI sub-cohort, are included in Additional file 1: Figures S1-S3.

\section{Male LUTS (including OAB) sub-cohort}

For monotherapy, mirabegron had the longest median TTD (205 days), followed by fesoterodine (115 days), trospium (102 days) and solifenacin (97 days) (Fig. 2a; Table 6). These drugs also displayed the highest 12-month persistence rates (Table 7). For combination therapies, tamsulosin plus trospium had the longest median TTD (144 days); however, the sample size was small $(n=65)$. The longest median TTD for combination therapy with a sample size $>100$ was 121 days for finasteride plus solifenacin plus tamsulosin (Fig. 2b). The highest 12-month persistence was with finasteride plus solifenacin (32.3\%) (Table 7).

\section{Male BPO sub-cohort}

For monotherapy, median TTD was longest for doxazosin and finasteride ( $>365$ days each [median not reached]), followed by tamsulosin (329 days) and dutasteride (305 days) (however, see sensitivity analyses below). Doxazosin had the highest 12 -month persistence $(67.8 \%)$ followed by finasteride (53.3\%) and tamsulosin (48.4\%). For combination therapies, the highest 12-month persistence was for dutasteride plus tamsulosin (56.3\%) followed by doxazosin plus dutasteride (55.6\%) (Additional file 1: Tables S8 and S9). 


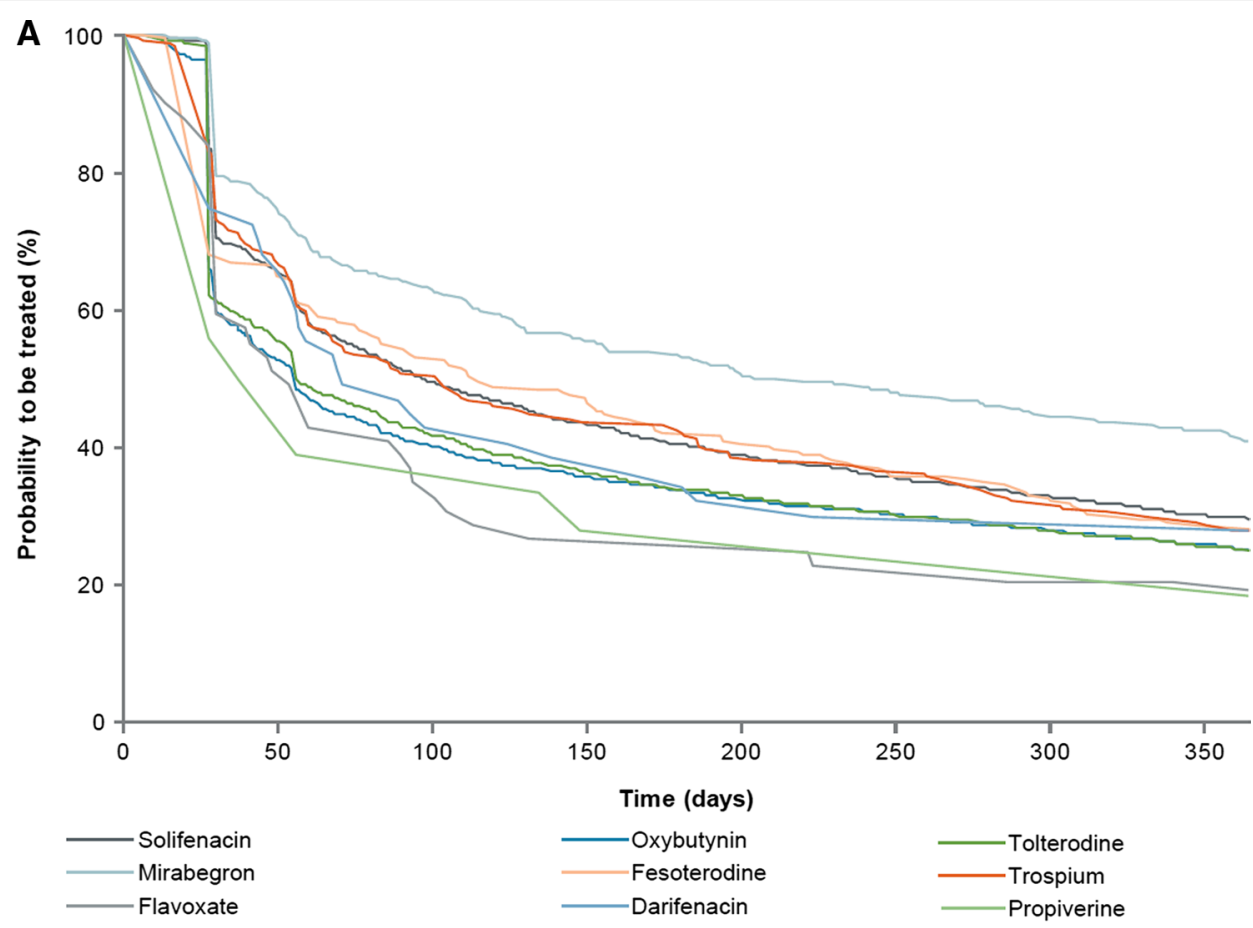

${ }^{\dagger}$ All monotherapies prescribed have been plotted.

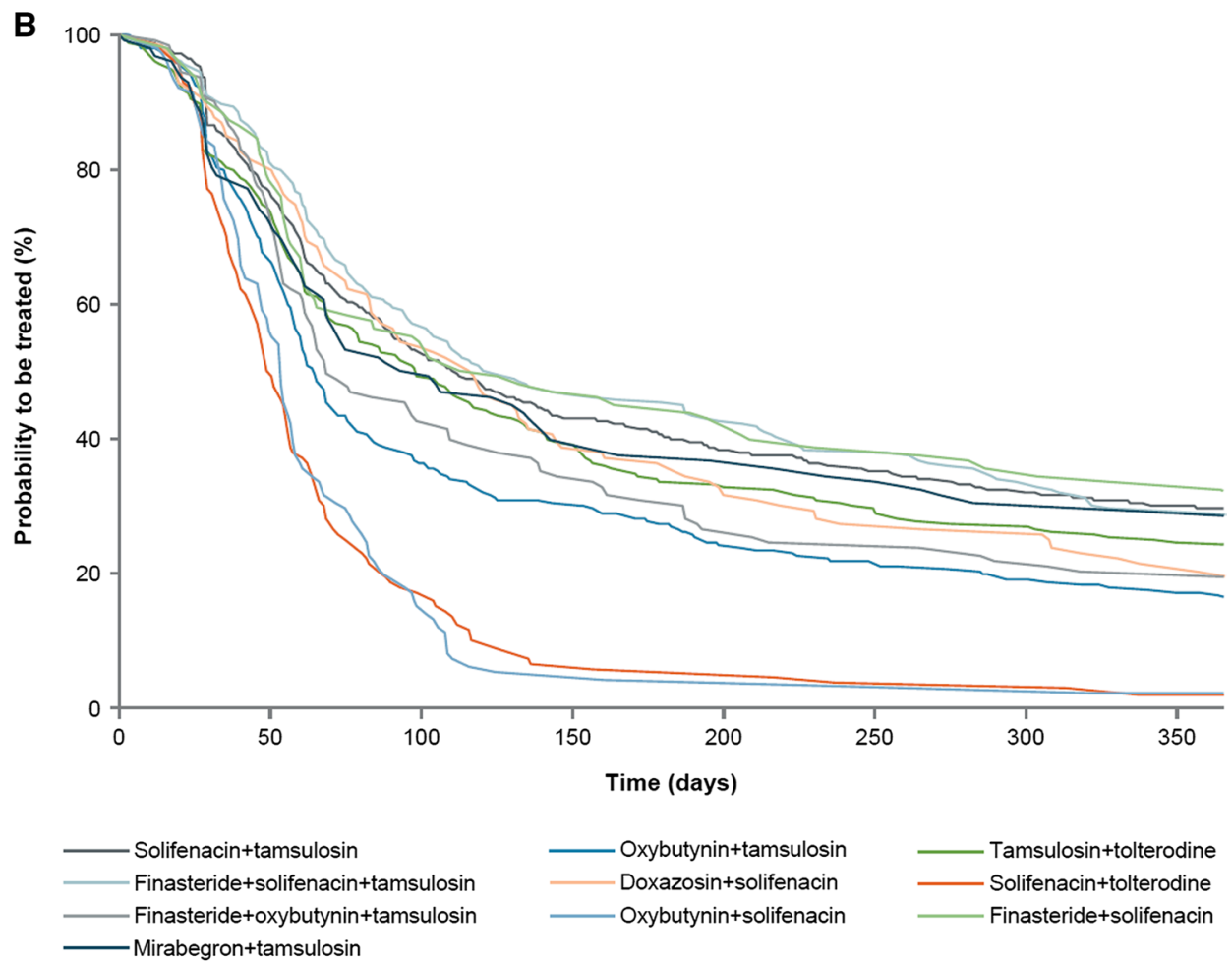

LUTS: lower urinary tract symptoms; $O A B$ : overactive bladder; TTD: time to discontinuation

${ }^{+}$The 10 most frequent combination drug therapies have been plotted.

Fig. 2 TTD in male LUTS (including OAB) sub-cohort (Kaplan-Meier estimates) ${ }^{\dagger}$ for $\mathbf{A}$ monotherapies. ${ }^{\dagger}$ All monotherapies prescribed have been plotted. B Combination drug therapies. LUTS: lower urinary tract symptoms; OAB: overactive bladder; TTD: time to discontinuation. ${ }^{\dagger}$ The 10 most frequent combination drug therapies have been plotted 
Table 6 Persistence in post-index period in the male LUTS (including $\mathrm{OAB}$ ) sub-cohort

\begin{tabular}{|c|c|c|}
\hline Index drug & $\mathrm{N}$ & $\begin{array}{l}\text { Persistence days } \\
\text { Median (Q1-Q3) }\end{array}$ \\
\hline \multicolumn{3}{|l|}{ Monotherapy } \\
\hline Solifenacin & 2759 & $97\left(30-365^{a}\right)$ \\
\hline Oxybutynin & 2613 & $56(28-363)$ \\
\hline Tolterodine & 1445 & $56(28-359)$ \\
\hline Mirabegron & 503 & $205\left(50-365^{a}\right)$ \\
\hline Fesoterodine & 285 & $115\left(28-365^{a}\right)$ \\
\hline Trospium & 227 & $102\left(30-365^{\mathrm{a}}\right)$ \\
\hline Flavoxate & 49 & $54(30-222)$ \\
\hline Darifenacin & 47 & $71\left(28-365^{\mathrm{a}}\right)$ \\
\hline Propiverine & 18 & $42(28-273)$ \\
\hline \multicolumn{3}{|l|}{ Combination drug therapy } \\
\hline Solifenacin + tamsulosin & 902 & $111\left(53-365^{a}\right)$ \\
\hline Oxybutynin + tamsulosin & 364 & $65(42-195)$ \\
\hline Tamsulosin + tolterodine & 248 & $99(48-335.5)$ \\
\hline Finasteride + solifenacin + tamsulosin & 233 & $121\left(61-365^{a}\right)$ \\
\hline Doxazosin + solifenacin & 114 & $117(59-308)$ \\
\hline Solifenacin + tolterodine & 114 & $50(34-75)$ \\
\hline Finasteride + oxybutynin + tamsulosin & 111 & $69(49-215)$ \\
\hline Oxybutynin + solifenacin & 102 & $54(38-82)$ \\
\hline Finasteride + solifenacin & 96 & $120\left(55-365^{a}\right)$ \\
\hline Mirabegron + tamsulosin & 96 & $98\left(47-365^{a}\right)$ \\
\hline Doxazosin + oxybutynin & 95 & $60(44-128)$ \\
\hline Mirabegron + solifenacin & 95 & $61(42-95)$ \\
\hline Fesoterodine + tamsulosin & 83 & $80(50-221)$ \\
\hline Dutasteride + solifenacin + tamsulosin & 79 & $74(51-235)$ \\
\hline Doxazosin + tolterodine & 69 & $95\left(50-365^{a}\right)$ \\
\hline Tamsulosin + trospium & 65 & $144\left(61-365^{a}\right)$ \\
\hline Finasteride + oxybutynin & 62 & $77(42-240)$ \\
\hline Finasteride + tamsulosin + tolterodine & 57 & $119\left(62-365^{a}\right)$ \\
\hline Alfuzosin + solifenacin & 47 & $124\left(42-365^{a}\right)$ \\
\hline Other combinations & 1405 & $63(41-151)$ \\
\hline
\end{tabular}

LUTS: lower urinary tract symptoms; $O A B$ : overactive bladder; $Q 1$ : lower quartile; Q3: upper quartile

${ }^{\mathrm{a}} \mathrm{Q} 3$ not reached by 365 days

\section{Female LUTS (including OAB) sub-cohort}

For monotherapy, median TTD (244 days) and 12-month persistence (43.5\%) was longest for mirabegron (Fig. 3a). Among combination therapies, persistence rates at 12 months were often not observable; however, the longest median TTD was 69 days for duloxetine plus tolterodine and 70 days for fesoterodine plus mirabegron (Fig. 3b, and Additional file 1: Tables S10 and S11).

\section{Female SUI sub-cohort}

For duloxetine, median TTD was 55 days and 12-month persistence rate was $22.0 \%$.

\section{Sensitivity analyses}

Results of sensitivity analyses were consistent with the main analyses, with one notable exception. In the male BPO sub-cohort, patients with a confirmed LUTS diagnosis had a lower median TTD on doxazosin (144 versus $>365$ days) and finasteride (170 versus $>365$ days) versus the main analysis (Additional file 1: Figures S4a and $b$ ). There were no notable findings in the other sensitivity analyses performed (Additional file 1: Table S12).

\section{Discussion}

This retrospective analysis in a UK GP primary care database complements previous UK studies of LUTS/ OAB and LUTS/BPO [20-22]. The study highlights the relatively low use of combination treatments that target OAB. Only a small proportion of LUTS (including OAB) patients were prescribed mirabegron with an antimuscarinic agent. However, use of this combination may have increased since the publication of studies such as BESIDE in 2016, SYNERGY in 2017 and SYNERGY II in 2018, which showed benefits of combination versus an antimuscarinic alone $[9,23,24]$. It was also notable that around 5\% each of men and women receiving OAB drugs were on a combination of two or more antimuscarinic agents (Tables 3 and 4), despite a lack of evidence supporting any benefit of this approach.

Our study also highlights the relatively low treatment rates for storage symptoms in men, despite the fact that these symptoms can be highly bothersome in men, even more so than voiding symptoms [25]. In the EPIC study, of all men identified with LUTS, over $80 \%$ had storage LUTS [10]. In the EpiLUTS study [13], storage symptoms were experienced by around two-thirds of men, with approximately $50 \%$ of men reporting mixed storage and voiding symptoms [13]. Alpha-blockers are the usual first-line treatment for men with LUTS suggestive of BPO $[6,26]$ and while they have been shown to relieve both voiding and storage symptoms [27], evidence suggests that up to two-thirds will not respond adequately to alpha-blocker monotherapy [14]. In these cases, the EAU guidelines recommend adding an OAB drug [6]. Therefore, it would be expected that the percentage of men who required $O A B$ treatment would be similar to the percentage of those with storage symptoms. This percentage is around $45 \%$ when calculated using data from the EpiLUTS study [13] — the percentage of LUTS patients with storage symptoms alone combined with around two thirds of LUTS patients with mixed symptoms (i.e., those who do not respond to first-line alpha-blocker therapy). However, in our study only around a quarter of all men being treated for LUTS received treatment specifically targeting storage symptoms (i.e., an antimuscarinic and/ or mirabegron alone or combined with a BPO drug), 
Table 7 Persistence at 1 month, 6 months and 1 year in male LUTS (including OAB) sub-cohort

\begin{tabular}{|c|c|c|c|c|c|}
\hline & $\mathbf{N}$ & 1 month $\%[95 \% \mathrm{Cl}]$ & 6 months $\%[95 \% \mathrm{Cl}]$ & 1 year $\%[95 \% \mathrm{Cl}]$ & $\begin{array}{l}\text { Median } \\
\text { (months) } \\
{[95 \% \mathrm{Cl}]}\end{array}$ \\
\hline \multirow[t]{2}{*}{ All } & 12,383 & 72.9 & 34.8 & 25.2 & 2.5 \\
\hline & & {$[72.1,73.6]$} & {$[33.9,35.6]$} & {$[24.4,26.0]$} & {$[2.4,2.7]$} \\
\hline Number of patients still at risk ${ }^{\mathrm{a}}$ & & 9024 & 4304 & 3105 & \\
\hline \multirow[t]{2}{*}{ Monotherapy } & 7946 & 65.6 & 37.6 & 27.7 & 2.5 \\
\hline & & {$[64.5,66.6]$} & {$[36.5,38.6]$} & {$[26.8,28.7]$} & {$[2.3,2.7]$} \\
\hline Number of patients still at risk ${ }^{\mathrm{a}}$ & & 5211 & 2986 & 2193 & \\
\hline \multirow[t]{2}{*}{ Combination drug therapy } & 4437 & 85.9 & 29.7 & 20.6 & 2.5 \\
\hline & & {$[84.9,86.9]$} & {$[28.4,31.1]$} & {$[19.4,21.8]$} & {$[2.4,2.7]$} \\
\hline Number of patients still at risk ${ }^{a}$ & & 3813 & 1318 & 912 & \\
\hline \multicolumn{6}{|l|}{ Monotherapy } \\
\hline \multirow[t]{2}{*}{ Darifenacin } & 47 & 74.5 & 34.0 & 27.7 & 2.3 \\
\hline & & {$[59.4,84.6]$} & {$[21.0,47.5]$} & {$[15.9,40.8]$} & {$[1.7,5.4]$} \\
\hline Number of patients still at risk ${ }^{\mathrm{a}}$ & & 35 & 16 & 13 & \\
\hline \multirow[t]{2}{*}{ Fesoterodine } & 285 & 67.4 & 41.8 & 28.1 & 3.8 \\
\hline & & {$[61.6,72.5]$} & {$[36.0,47.4]$} & {$[23.0,33.4]$} & {$[2.7,5.2]$} \\
\hline Number of patients still at risk ${ }^{\mathrm{a}}$ & & 192 & 119 & 80 & \\
\hline \multirow[t]{2}{*}{ Flavoxate } & 49 & 59.2 & 26.5 & 20.4 & 1.8 \\
\hline & & {$[44.2,71.4]$} & {$[15.2,39.3]$} & {$[10.5,32.6]$} & {$[1.0,3.1]$} \\
\hline Number of patients still at risk ${ }^{a}$ & & 29 & 13 & 9 & \\
\hline \multirow[t]{2}{*}{ Mirabegron } & 503 & 79.5 & 53.1 & 40.9 & 6.7 \\
\hline & & {$[75.7,82.8]$} & {$[48.6,57.3]$} & {$[36.6,45.2]$} & {$[5.2,9.5]$} \\
\hline Number of patients still at risk ${ }^{\mathrm{a}}$ & & 400 & 267 & 204 & \\
\hline \multirow[t]{2}{*}{ Oxybutynin } & 2613 & 59.6 & 33.5 & 25.1 & 1.8 \\
\hline & & {$[57.7,61.4]$} & {$[31.7,35.3]$} & {$[23.5,26.8]$} & {$[1.7,1.9]$} \\
\hline Number of patients still at risk ${ }^{a}$ & & 1557 & 875 & 653 & \\
\hline \multirow[t]{2}{*}{ Propiverine } & 18 & 55.6 & 27.8 & 22.2 & 1.4 \\
\hline & & {$[30.5,74.8]$} & {$[10.1,48.9]$} & {$[6.9,42.9]$} & {$[0.9,4.9]$} \\
\hline Number of patients still at risk ${ }^{a}$ & & 10 & 5 & 4 & \\
\hline \multirow[t]{2}{*}{ Solifenacin } & 2759 & 70.2 & 40.2 & 29.5 & 3.2 \\
\hline & & {$[68.5,71.9]$} & {$[38.4,42.1]$} & {$[27.8,31.2]$} & {$[2.9,3.6]$} \\
\hline Number of patients still at risk ${ }^{\mathrm{a}}$ & & 1938 & 1110 & 812 & \\
\hline \multirow[t]{2}{*}{ Tolterodine } & 1445 & 61.2 & 33.7 & 24.8 & 1.8 \\
\hline & & {$[58.6,63.6]$} & {$[31.3,36.1]$} & {$[22.6,27.1]$} & {$[1.8,2.2]$} \\
\hline Number of patients still at risk ${ }^{a}$ & & 884 & 487 & 355 & \\
\hline \multirow[t]{2}{*}{ Trospium } & 227 & 73.1 & 41.4 & 27.8 & 3.4 \\
\hline & & {$[66.9,78.4]$} & {$[35.0,47.7]$} & {$[22.1,33.7]$} & {$[2.2,4.9]$} \\
\hline Number of patients still at risk ${ }^{a}$ & & 166 & 94 & 63 & \\
\hline \multicolumn{6}{|l|}{ Combination drug therapy } \\
\hline \multirow[t]{2}{*}{ Alfuzosin + solifenacin } & 47 & 87.2 & 40.4 & 29.8 & 4.1 \\
\hline & & {$[73.8,94.1]$} & {$[26.5,53.9]$} & {$[17.6,43.0]$} & {$[2.1,6.9]$} \\
\hline Number of patients still at risk ${ }^{a}$ & & 41 & 19 & 14 & \\
\hline \multirow[t]{2}{*}{ Doxazosin + oxybutynin } & 95 & 87.4 & 18.9 & NO & 2 \\
\hline & & {$[78.8,92.6]$} & {$[11.8,27.4]$} & - & {$[1.8,2.3]$} \\
\hline Number of patients still at risk ${ }^{a}$ & & 83 & 18 & 13 & \\
\hline \multirow[t]{2}{*}{ Doxazosin + solifenacin } & 114 & 89.5 & 36.0 & 20.2 & 3.8 \\
\hline & & {$[82.2,93.9]$} & {$[27.3,44.7]$} & {$[13.4,28.0]$} & {$[2.8,4.7]$} \\
\hline Number of patients still at risk & & 102 & 41 & 23 & \\
\hline Doxazosin + tolterodine & 69 & 87.0 & 39.1 & 29.0 & 3.1 \\
\hline
\end{tabular}


Table 7 (continued)

\begin{tabular}{|c|c|c|c|c|c|}
\hline & $\mathbf{N}$ & 1 month \% $[95 \% \mathrm{Cl}]$ & 6 months $\%[95 \% \mathrm{Cl}]$ & 1 year \% $[95 \% \mathrm{Cl}]$ & $\begin{array}{l}\begin{array}{l}\text { Median } \\
\text { (months) }\end{array} \\
{[95 \% \mathrm{Cl}]}\end{array}$ \\
\hline & & {$[76.4,93.0]$} & {$[27.7,50.4]$} & {$[18.8,39.9]$} & {$[2.1,6.4]$} \\
\hline Number of patients still at risk ${ }^{a}$ & & 60 & 27 & 20 & \\
\hline \multirow[t]{2}{*}{ Dutasteride + solifenacin + tamsulosin } & 79 & 89.9 & 27.8 & 21.5 & 2.4 \\
\hline & & {$[80.8,94.8]$} & {$[18.5,38.0]$} & {$[13.3,31.1]$} & {$[2.1,3.6]$} \\
\hline Number of patients still at risk ${ }^{a}$ & & 71 & 22 & 17 & \\
\hline \multirow[t]{2}{*}{ Fesoterodine + tamsulosin } & 83 & 85.5 & 28.9 & NO & 2.6 \\
\hline & & {$[75.9,91.5]$} & {$[19.6,38.9]$} & - & {$[2.1,3.3]$} \\
\hline Number of patients still at risk ${ }^{a}$ & & 71 & 24 & 12 & \\
\hline \multirow[t]{2}{*}{ Finasteride + oxybutynin } & 62 & 88.7 & 32.3 & 17.7 & 2.5 \\
\hline & & {$[77.8,94.5]$} & {$[21.1,43.9]$} & {$[9.5,28.1]$} & {$[1.8,3.6]$} \\
\hline Number of patients still at risk ${ }^{\mathrm{a}}$ & & 55 & 20 & 11 & \\
\hline \multirow[t]{2}{*}{ Finasteride + oxybutynin + tamsulosin } & 111 & 91.0 & 30.6 & 19.8 & 2.3 \\
\hline & & {$[83.9,95.0]$} & {$[22.3,39.3]$} & {$[13.0,27.7]$} & {$[2.0,3.6]$} \\
\hline Number of patients still at risk ${ }^{a}$ & & 101 & 34 & 22 & \\
\hline \multirow{2}{*}{ Finasteride + solifenacin } & 96 & 90.6 & 44.8 & 32.3 & 3.9 \\
\hline & & {$[82.8,95.0]$} & {$[34.7,54.4]$} & {$[23.2,41.7]$} & {$[2.1,6.9]$} \\
\hline Number of patients still at risk ${ }^{a}$ & & 87 & 43 & 31 & \\
\hline \multirow[t]{2}{*}{ Finasteride + solifenacin + tamsulosin } & 233 & 91.0 & 45.1 & 28.8 & 4 \\
\hline & & {$[86.5,94.0]$} & {$[38.6,51.3]$} & {$[23.1,34.7]$} & {$[3.4,6.2]$} \\
\hline Number of patients still at risk ${ }^{\mathrm{a}}$ & & 212 & 105 & 67 & \\
\hline \multirow[t]{2}{*}{ Finasteride + tamsulosin + tolterodine } & 57 & 96.5 & 40.4 & 28.1 & 3.9 \\
\hline & & {$[86.7,99.1]$} & {$[27.7,52.7]$} & {$[17.2,40.0]$} & {$[2.8,6.7]$} \\
\hline Number of patients still at risk ${ }^{\mathrm{a}}$ & & 55 & 23 & 15 & \\
\hline \multirow[t]{2}{*}{ Finasteride + tolterodine } & 41 & 85.4 & 29.3 & 22.0 & 1.7 \\
\hline & & {$[70.3,93.1]$} & {$[16.4,43.4]$} & {$[10.9,35.5]$} & {$[1.5,3.9]$} \\
\hline Number of patients still at risk ${ }^{a}$ & & 35 & 12 & 9 & \\
\hline \multirow[t]{2}{*}{ Mirabegron + solifenacin } & 95 & 85.3 & NO & NO & 2 \\
\hline & & {$[76.4,91.0]$} & - & - & {$[1.7,2.5]$} \\
\hline Number of patients still at risk ${ }^{a}$ & & 81 & 11 & 6 & \\
\hline \multirow{2}{*}{ Mirabegron + tamsulosin } & 96 & 82.3 & 37.5 & 29.2 & 3.2 \\
\hline & & {$[73.1,88.6]$} & {$[27.9,47.1]$} & {$[20.5,38.4]$} & {$[2.2,4.7]$} \\
\hline Number of patients still at risk & & 79 & 36 & 28 & \\
\hline \multirow[t]{2}{*}{ Oxybutynin + solifenacin } & 102 & 84.3 & NO & NO & 1.8 \\
\hline & & {$[75.7,90.1]$} & - & - & {$[1.6,1.9]$} \\
\hline Number of patients still at risk ${ }^{a}$ & & 86 & 4 & 2 & \\
\hline \multirow[t]{2}{*}{ Oxybutynin + tamsulosin } & 364 & 82.4 & 26.9 & 16.5 & 2.1 \\
\hline & & {$[78.1,86.0]$} & {$[22.5,31.6]$} & {$[12.9,20.5]$} & {$[2.0,2.3]$} \\
\hline Number of patients still at risk ${ }^{a}$ & & 300 & 98 & 60 & \\
\hline \multirow[t]{2}{*}{ Solifenacin + tamsulosin } & 902 & 86.7 & 39.9 & 29.4 & 3.6 \\
\hline & & {$[84.3,88.7]$} & {$[36.7,43.1]$} & {$[26.4,32.4]$} & {$[3.2,4.1]$} \\
\hline Number of patients still at risk ${ }^{a}$ & & 782 & 360 & 264 & \\
\hline \multirow[t]{2}{*}{ Solifenacin + tolterodine } & 114 & 77.2 & NO & NO & 1.6 \\
\hline & & {$[68.3,83.9]$} & - & - & {$[1.5,1.8]$} \\
\hline Number of patients still at risk ${ }^{a}$ & & 88 & 6 & 2 & \\
\hline \multirow[t]{2}{*}{ Tamsulosin + tolterodine } & 248 & 82.3 & 33.5 & 24.2 & 3.3 \\
\hline & & {$[76.9,86.5]$} & {$[27.7,39.4]$} & {$[19.1,29.7]$} & {$[2.6,4.0]$} \\
\hline Number of patients still at risk ${ }^{\mathrm{a}}$ & & 204 & 83 & 60 & \\
\hline Tamsulosin + trospium & 65 & 90.8 & 41.5 & 29.2 & 4.7 \\
\hline
\end{tabular}


Table 7 (continued)

\begin{tabular}{|c|c|c|c|c|c|}
\hline & $\mathrm{N}$ & 1 month $\%[95 \% \mathrm{Cl}]$ & 6 months $\%[95 \% \mathrm{Cl}]$ & 1 year $\%[95 \% \mathrm{Cl}]$ & $\begin{array}{l}\text { Median } \\
\text { (months) } \\
{[95 \% \mathrm{Cl}]}\end{array}$ \\
\hline & & {$[80.6,95.7]$} & {$[29.5,53.1]$} & {$[18.8,40.5]$} & {$[2.9,7.1]$} \\
\hline Number of patients still at risk ${ }^{a}$ & & 59 & 27 & 19 & \\
\hline \multirow[t]{2}{*}{ Other combinations } & 1364 & 85.1 & 22.4 & 15.9 & 2.1 \\
\hline & & {$[83.1,86.9]$} & {$[20.2,24.6]$} & {$[14.0,17.9]$} & {$[2.0,2.2]$} \\
\hline Number of patients still at risk ${ }^{a}$ & & 1161 & 305 & 217 & \\
\hline
\end{tabular}

Not Observable indicates that the number of patients still at risk was below the $20 \%$ of the initial sample threshold required to calculate persistence, or the median was not reached

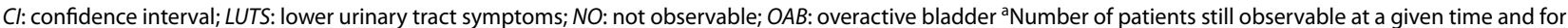
whom no events occurred

although this figure refers only to men with treated LUTS, in contrast to the other studies which were based on the general population. Thus, some men with storage symptoms may be receiving inadequate treatment in clinical practice, despite storage symptoms often being the most bothersome component of LUTS [28].

We might also expect to see OAB/BPO drug combination therapy in up to one-third of all men being treated for LUTS (i.e., two-thirds of those with mixed symptoms) $[13,14]$. However, in the current study, only $7 \%$ were receiving alpha-blocker plus antimuscarinic combination treatment therapy (and only $8 \%$ were on any OAB/BPO drug combination), which is consistent with another UK study in which $15 \%$ of men with mixed LUTS were reported to be receiving an alpha-blocker combined with an antimuscarinic agent [20].

As well as alpha-blockers and 5-ARIs, the EAU guidelines recommend the use of phosphodiesterase type 5 inhibitors (PDE5Is) for the treatment of men with moderate-to-severe LUTS with or without erectile dysfunction [6]. Tadalafil, the only currently licensed PDE5I for male LUTS [6], has been shown to relieve both voiding and storage symptoms [29], although additional therapy may be required for patients with severe LUTS related to BOO [30]. However, this study did not look at tadalafil use due to potential misclassification of patients receiving the drug for erectile dysfunction.

The reasons for the low treatment of storage symptoms in men may be historical, reflecting overemphasis on the prostate-related component of LUTS rather than bladder-related issues. Furthermore, there may be a perceived risk of precipitating urinary retention when using bladder antimuscarinic agents in men with evidence of obstruction, although the available evidence suggests that this risk is low [31]. There is already good evidence supporting the use of alpha-blocker/antimuscarinic combination therapy in men with mixed symptoms [16, 17]. More recently, two randomized, placebo-controlled trials have also demonstrated that mirabegron add-on therapy in men who have residual OAB symptoms while being treated with tamsulosin for LUTS is both effective and well-tolerated $[18,32]$. It is hoped that this new evidence will help to improve the overall management of men with mixed symptoms.

Antimuscarinic agents and beta- 3 agonists are recommended first-line pharmacological treatments for both men and women with $\mathrm{OAB}[7,8]$ and men with moderate-to-severe LUTS with predominant bladder storage symptoms [6]. However, with antimuscarinics, long-term persistence is often poor due to unmet treatment expectations or adverse events [33]. In our study, mirabegron monotherapy had the highest persistence (both in men and women). Several observational studies also reported higher persistence with mirabegron vs antimuscarinics $[22,34]$. Persistence was greater with drug monotherapy than in combination drug therapy, and was particularly poor with combinations of two antimuscarinics in both men and women.

For monotherapy targeting BPO and voiding symptoms (e.g. alpha-blockers and 5-ARIs), persistence was highest for doxazosin and finasteride, but this was not evident in sensitivity analyses based on confirmed LUTS diagnosis. This suggests that the higher persistence with these agents in the main sub-cohorts may be driven by their use in other disorders (e.g., doxazosin for hypertension) and it is notable that only $3 \%$ of patients on doxazosin had a LUTS diagnostic code.

A limitation of our study is that in CPRD GOLD, GPs do not systematically report prescriptions issued in secondary care, and reasons for discontinuation were not available in CPRD, which limits interpretation of persistence results. In addition, some treatments are prescribed for conditions other than OAB, LUTS, BPO or SUI (e.g., doxazosin, finasteride and duloxetine), which may influence some of the treatment pattern and/or persistence estimates. The inclusion of fixed-dose combinations may 


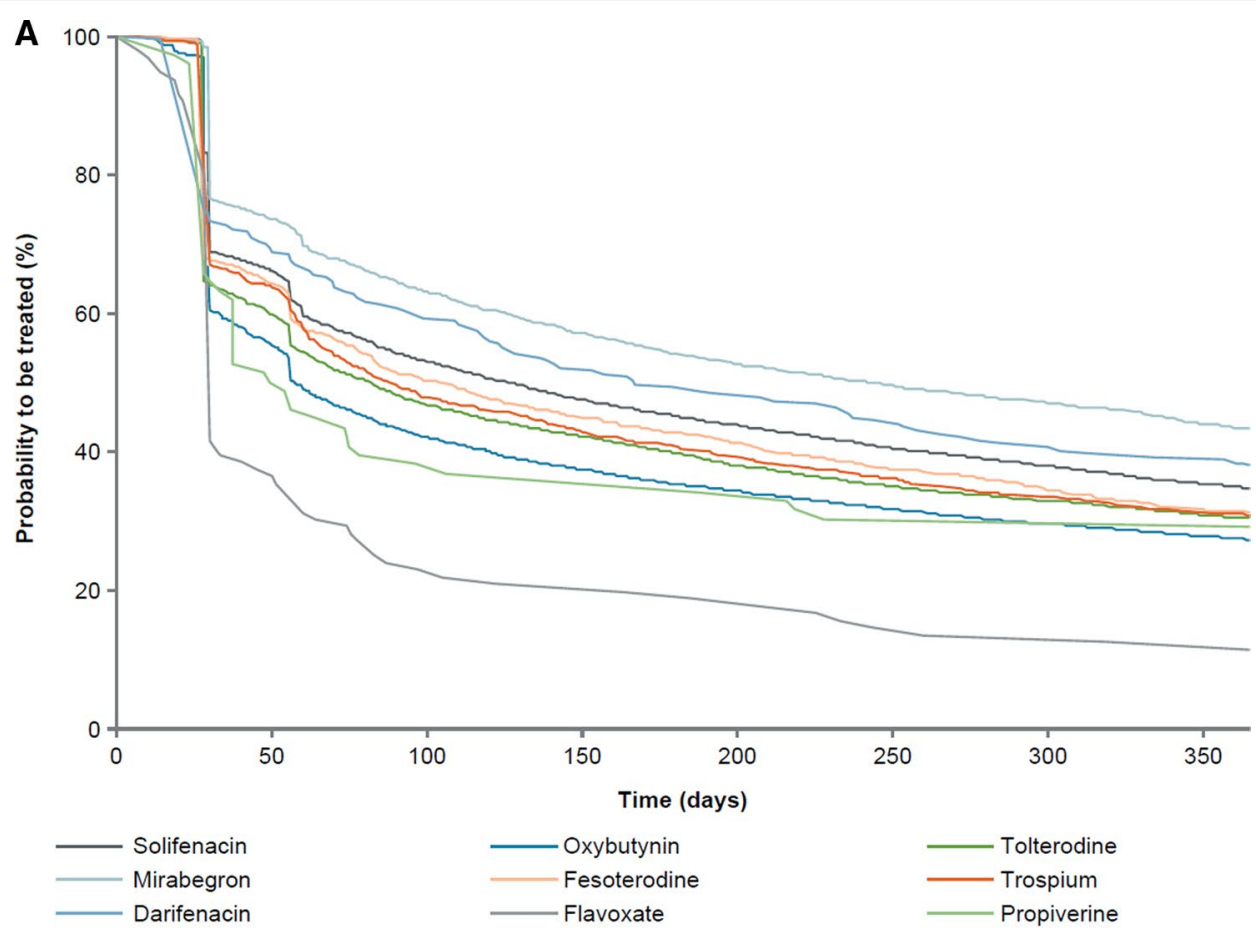

${ }^{\dagger}$ All monotherapies prescribed have been plotted.

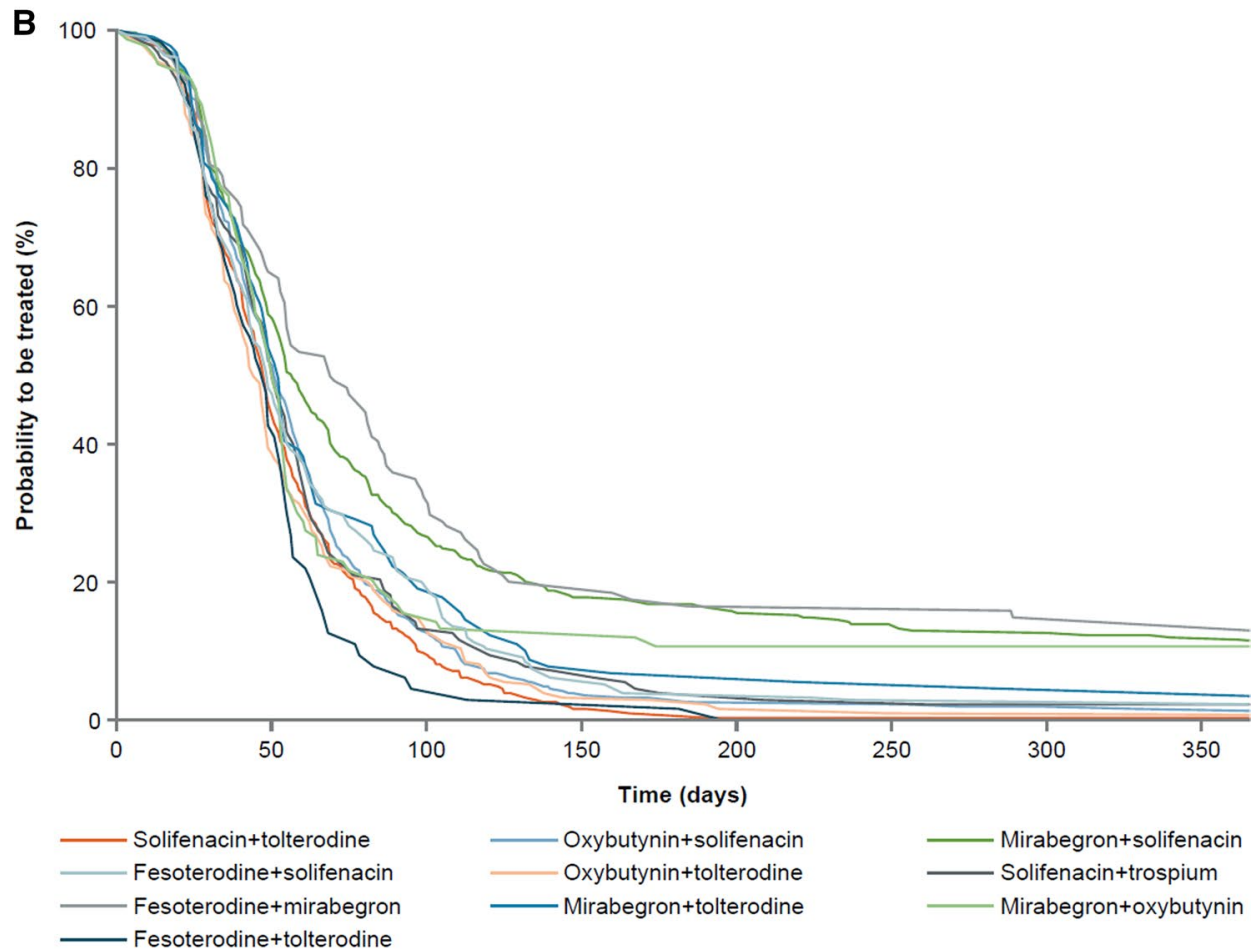

LUTS: lower urinary tract symptoms; OAB: overactive bladder; TTD: time to discontinuation

${ }^{\dagger}$ The 10 most frequent combination drug therapies have been plotted.

Fig. 3 TTD in female LUTS (including OAB) sub-cohort (Kaplan-Meier estimates) ${ }^{\dagger}$ for $\mathbf{A}$ monotherapies. ${ }^{\dagger}$ All monotherapies prescribed have been plotted. B Combination drug therapies. LUTS: lower urinary tract symptoms; OAB: overactive bladder; TTD: time to discontinuation. ${ }^{\dagger}$ The 10 most frequent combination drug therapies have been plotted 
increase the overall persistence with tamsulosin/solifenacin combination therapy [35]; for tamsulosin/dutasteride fixed-dose combination, the available evidence suggests it may have no impact on persistence [36]. Finally, as this study was performed using a UK general practice database, it is unclear to what extent the results would be generalizable to other healthcare systems.

\section{Conclusions}

This study provides new real-world evidence suggesting that men with LUTS may be under-treated with pharmacotherapies that specifically target storage symptoms. Only around a quarter of the men being treated for any LUTS received treatment specifically targeting storage symptoms and around $8 \%$ of men received a combination of $\mathrm{BPO} / \mathrm{OAB}$ drugs for mixed symptoms. In addition, use of combination $\mathrm{OAB}$ treatment was low in both men and women, which may reflect the lack of evidence for this approach at the time these patients were being treated. Of all OAB medications, numerically the highest rates of monotherapy persistence were seen with mirabegron in both men and women. Persistence was worse when using combination drug therapy and particularly poor when using two antimuscarinic agents. By highlighting the possible under-treatment of men with treatments that target storage symptoms and the low use of combination $\mathrm{OAB}$ treatment (especially with mirabegron plus an antimuscarinic agent), this may help clinicians in the UK to reassess their approach to pharmacotherapy for patients with bothersome LUTS.

\begin{abstract}
Abbreviations
5-ARI: 5-Alpha reductase inhibitor; BNF: British National Formulary; BOO: Bladder outlet obstruction; BPO: Benign prostatic obstruction; $\mathrm{Cl}$ : Confidence interval; CPRD: Clinical Practice Research Datalink; EAU: European Association of Urology; GP: General practitioner; IMD: Index of Multiple Deprivation; LUTS: Lower urinary tract symptoms; MAGD: Maximum allowable gap duration; NO: Not observable; NRES: National Research Ethics Service Committee; OAB: Overactive bladder; PDE5I: Phosphodiesterase type 5 inhibitor; Q1: Lower quartile; Q3: Upper quartile; QOF: Quality and Outcomes Framework; SA: Sensitivity analysis; SD: Standard deviation; SUI: Stress urinary incontinence; TTD: Time to discontinuation.
\end{abstract}

\section{Supplementary Information}

The online version contains supplementary material available at https://doi. org/10.1186/s12894-021-00881-w.

Additional file 1. Supplementary Tables 1 to 12 and Supplementary Figures 1 to 4

\section{Acknowledgements}

Medical writing support was provided by Hayley Owen and Tom Lavelle (Bioscript Medical Ltd, Macclesfield, UK).

\section{Authors' contributions}

Conception and design of study: MA, ML, PJOC, NC, AJ, FF and RvM. Data acquisition: MA and ML. Data analysis and interpretation: MA, ML, PJOC, NC, AJ, FF and RvM. Statistical analysis: MA and ML. Obtaining funding: MA, ML, NC, AJ and FF. Administrative, technical or material support: MA, ML, AJ and FF. Supervision: MA, ML and FF. Study report preparation, review and finalization process: MA and ML. Critical revision of the publication for intellectual content: MA, ML, PJOC, AJ, FF and RvM. All authors read and approved the final manuscript.

\section{Funding}

This study was funded by Astellas Pharma Inc. and Innovate UK, and included a knowledge transfer partnership (KTP) between Manchester Metropolitan University and Astellas Pharma Inc. The KTP involved a collaboration between Manchester Metropolitan University and Astellas Pharma Inc. who had joint roles in: the design and conduct of the study; management, analysis and interpretation of the data; preparation, review and approval of the manuscript. Medical writing/editorial support was provided by Hayley Owen and Tom Lavelle from Bioscript Medical Ltd, Macclesfield, UK and funded by the study sponsor.

\section{Availability of data and materials}

Researchers may request access to anonymized participant level data, trial level data and protocols from Astellas sponsored clinical trials at www.clini calstudydatarequest.com. For the Astellas criteria on data sharing see: https:// clinicalstudydatarequest.com/Study-Sponsors/Study-Sponsors-Astellas.aspx.

\section{Declarations}

\section{Ethical approval and consent to participate}

The CPRD has obtained ethical approval from a National Research Ethics Service Committee (NRES), for all purely observational research using anonymized CPRD data; namely, studies which do not include patient involvement. The independent scientific advisory committee is responsible for reviewing protocols for scientific quality, but may recommend that study-specific ethical approval is sought if ethical issues arise in relation to an individual study. This study met the requirements under the CPRD obtained ethical approval from NRES. Only de-identified data were obtained, and patients could opt out if they did not wish to have their data used for research purposes.

\section{Consent for publication}

Not applicable.

\section{Competing interests}

MA, ML and PJOC are employees of Astellas Pharma Europe Ltd.; NC was an employee of Astellas Pharma Europe Ltd. when the manuscript was written; MA and AJ were working as associates as part of the Knowledge Transfer Partnership (KTP) between Astellas and Manchester Metropolitan University during the time this research was carried out; FF received grant funding from Astellas Pharma during the conduct of the study; RvM was an employee of Astellas Global Development, Leiden at the time of this work.

\section{Author details}

${ }^{1}$ Manchester Metropolitan University, Manchester, UK. ${ }^{2}$ Present Address: Astellas Pharma Europe Ltd, Addlestone, UK. ${ }^{3}$ Astellas Global Development, Leiden, The Netherlands.

Received: 19 February 2021 Accepted: 11 August 2021

Published online: 02 September 2021

\section{References}

1. Abrams P, Cardozo L, Fall M, Griffiths D, Rosier P, Ulmsten U, van Kerrebroeck $P$, Victor A, Wein A. The standardisation of terminology of lower urinary tract function: report from the Standardisation Subcommittee of the International Continence Society. Neurourol Urodyn. 2002:21(2):167-78 
2. D'Ancona C, Haylen B, Oelke M, Abranches-Monteiro L, Arnold E, Goldman $\mathrm{H}$, Hamid R, Homma Y, Marcelissen T, Rademakers K, et al. The International Continence Society (ICS) report on the terminology for adult male lower urinary tract and pelvic floor symptoms and dysfunction. Neurourol Urodyn. 2019;38(2):433-77.

3. Haylen BT, de Ridder D, Freeman RM, Swift SE, Berghmans B, Lee J, Monga $A$, Petri E, Rizk DE, Sand PK, et al. An International Urogynecological Association (IUGA)/International Continence Society (ICS) joint report on the terminology for female pelvic floor dysfunction. Neurourol Urodyn. 2010;29(1):4-20.

4. International Continence Society: ICS Glossary - Overactive bladder (OAB, urgency) syndrome. https://www.ics.org/glossary/symptom/overactive bladderoaburgencysyndrome. Accessed June 2021.

5. DeMaagd GA, Davenport TC. Management of urinary incontinence. PT. 2012;37(6):345-361H.

6. Gravas S, Cornu JN, Gacci M, Gratzke C, Herrmann TRW, Mamoulakis C, Rieken M, Speakman MJ, Tikkinen KAO, Guidelines Associates et al. EAU guidelines on management of non-neurogenic male lower urinary tract symptoms (LUTS), incl. benign prostatic obstruction (BPO). https:// uroweb.org/quideline/treatment-of-non-neurogenic-male-luts/. Accessed May 2021.

7. Burkhard FC, Bosch JLHR, Cruz F, Lemack GE, Nambiar AK, Thiruchelvam N, Tubaro A, Associates: G, Ambühl D, Bedretdinova DA et al. EAU guidelines on urinary incontinence. https://uroweb.org/guideline/urina ry-incontinence/. Accessed December 2019.

8. Gormley EA, Lightner DJ, Burgio KL, Chai TC, Clemens JQ, Culkin DJ, Das AK, Foster HE, Jr., Scarpero HM, Tessier CD et al. Diagnosis and treatment of non-neurogenic overactive bladder (OAB) in adults: an AUA/SUFU guideline (2019). https://www.auanet.org/guidelines/overactive-bladd er-(oab)-guideline\#×2903. Accessed October 2019.

9. Gratzke C, van Maanen R, Chapple C, Abrams P, Herschorn S, Robinson D, Ridder A, Stoelzel M, Paireddy A, Yoon SJ, et al. Long-term safety and efficacy of mirabegron and solifenacin in combination compared with monotherapy in patients with overactive bladder: a randomised, multicentre phase 3 study (SYNERGY II). Eur Urol. 2018;74(4):501-9.

10. Irwin DE, Milsom I, Hunskaar S, Reilly K, Kopp Z, Herschorn S, Coyne K, Kelleher C, Hampel C, Artibani W, et al. Population-based survey of urinary incontinence, overactive bladder, and other lower urinary tract symptoms in five countries: results of the EPIC study. Eur Urol. 2006;50(6):1306-14 (discussion 1314-1315).

11. de Mey C. alpha(1)-blockers for BPH: are there differences? Eur Urol. 1999;36(Suppl 3):52-63.

12. McVary KT, Roehrborn CG, Avins AL, Barry MJ, Bruskewitz RC, Donnell RF, Foster HE Jr, Gonzalez CM, Kaplan SA, Penson DF, et al. Update on AUA guideline on the management of benign prostatic hyperplasia. J Urol. 2011;185(5):1793-803.

13. Sexton CC, Coyne KS, Kopp ZS, Irwin DE, Milsom I, Aiyer LP, Tubaro A, Chapple CR, Wein AJ. EpiLUTS Team: The overlap of storage, voiding and postmicturition symptoms and implications for treatment seeking in the USA, UK and Sweden: EpiLUTS. BJU Int. 2009;103(Suppl 3):12-23.

14. Lee HN, Lee KS, Kim JC, Chung BH, Kim CS, Lee JG, Kim DK, Park CH, Park JK, Hong SJ. Rate and associated factors of solifenacin add-on after tamsulosin monotherapy in men with voiding and storage lower urinary tract symptoms. Int J Clin Pract. 2015;69(4):444-53.

15. Filson CP, Hollingsworth JM, Clemens JQ, Wei JT. The efficacy and safety of combined therapy with alpha-blockers and anticholinergics for men with benign prostatic hyperplasia: a meta-analysis. J Urol. 2013;190(6):2153-60.

16. van Kerrebroeck P, Chapple C, DrogendijkT, Klaver M, Sokol R, Speakman M, Traudtner K, Drake MJ, Group NS. Combination therapy with solifenacin and tamsulosin oral controlled absorption system in a single tablet for lower urinary tract symptoms in men: efficacy and safety results from the randomised controlled NEPTUNE trial. Eur Urol. 2013;64(6):1003-12.

17. Rees J, Foley S, Huang M, Rosa Arias J, Skoumal R, Walters C, Yavuz Y, De Wachter $\mathrm{S}$. Vesomni improves the quality of life in men with lower urinary tract symptoms in routine clinical practice in Europe. Neurourol Urodyn. 2019:38(3):981-9.

18. Kaplan SA, Herschorn S, McVary KT, Staskin D, Chapple C, Foley S, Cambronero Santos J, Kristy RM, Choudhury N, Hairston J, et al. Efficacy and safety of mirabegron versus placebo add-on therapy in men with overactive bladder symptoms receiving tamsulosin for underlying benign prostatic hyperplasia: a randomized, phase 4 study (PLUS). J Urol. 2020:203(6):1163-71

19. Herrett E, Gallagher AM, Bhaskaran K, Forbes H, Mathur R, van Staa T, Smeeth L. Data resource profile: Clinical Practice Research Datalink (CPRD). Int J Epidemiol. 2015;44(3):827-36.

20. Hakimi Z, Johnson M, Nazir J, Blak B, Odeyemi IA. Drug treatment patterns for the management of men with lower urinary tract symptoms associated with benign prostatic hyperplasia who have both storage and voiding symptoms: a study using the health improvement network UK primary care data. Curr Med Res Opin. 2015;31(1):43-50.

21. Wagg A, Compion G, Fahey A, Siddiqui E. Persistence with prescribed antimuscarinic therapy for overactive bladder: a UK experience. BJU Int. 2012;110(11):1767-74.

22. Chapple CR, Nazir J, Hakimi Z, Bowditch S, Fatoye F, Guelfucci F, Khemiri A, Siddiqui E, Wagg A. Persistence and adherence with mirabegron versus antimuscarinic agents in patients with overactive bladder: a retrospective observational study in UK clinical practice. Eur Urol. 2017;72(3):389-99.

23. Drake MJ, Chapple C, Esen AA, Athanasiou S, Cambronero J, Mitcheson D, Herschorn S, Saleem T, Huang M, Siddiqui E, et al. Efficacy and safety of mirabegron add-on therapy to solifenacin in incontinent overactive bladder patients with an inadequate response to initial 4-week solifenacin monotherapy: a randomised double-blind multicentre phase $3 \mathrm{~B}$ study (BESIDE). Eur Urol. 2016;70(1):136-45.

24. Herschorn S, Chapple CR, Abrams P, Arlandis S, Mitcheson D, Lee KS, Ridder A, Stoelzel M, Paireddy A, van Maanen R, et al. Efficacy and safety of combinations of mirabegron and solifenacin compared with monotherapy and placebo in patients with overactive bladder (SYNERGY study). BJU Int. 2017;120(4):562-75.

25. Agarwal A, Eryuzlu LN, Cartwright R, Thorlund K, Tammela TL, Guyatt GH, Auvinen A, Tikkinen KA. What is the most bothersome lower urinary tract symptom? Individual- and population-level perspectives for both men and women. Eur Urol. 2014;65(6):1211-7.

26. Rees J, Bultitude M, Challacombe B. The management of lower urinary tract symptoms in men. BMJ. 2014;348:e3861.

27. Chapple CR, Wyndaele JJ, Nordling J, Boeminghaus F, Ypma AF, Abrams $P$, on behalf of the European Tamsulosin Study Group. Tamsulosin, the first prostate-selective a1 A-adrenoceptor antagonist. A meta-analysis of two randomized, placebo-controlled, multicentre studies in patients with benign prostatic obstruction (symptomatic BPH). Eur Urol. 1996;29(2):155-67.

28. Peters TJ, Donovan JL, Kay HE, Abrams P, de la Rosette JJ, Porru D, Thuroff JW. The International Continence Society "Benign Prostatic Hyperplasia" Study: the botherosomeness of urinary symptoms. J Urol. 1997;157(3):885-9.

29. Matsukawa Y, Majima T, Matsuo K, Funahashi Y, Kato M, Yamamoto T, Gotoh M. Effects of tadalafil on storage and voiding function in patients with male lower urinary tract symptoms suggestive of benign prostatic hyperplasia: a urodynamic-based study. Int J Urol. 2018;25(3):246-50.

30. Sebastianelli A, Spatafora P, Morselli S, Vignozzi L, Serni S, McVary KT, Kaplan S, Gravas S, Chapple C, Gacci M. Tadalafil alone or in combination with tamsulosin for the management for LUTS/BPH and ED. Curr Urol Rep. 2020;21(12):56.

31. Oelke M, Speakman MJ, Desgrandchamps F, Mamoulakis C. Acute urinary retention rates in the general male population and in adult men with lower urinary tract symptoms participating in pharmacotherapy trials: a literature review. Urology. 2015;86(4):654-65.

32. Kakizaki H, Lee KS, Yamamoto O, Jong JJ, Katou D, Sumarsono B, Uno S, Yamaguchi O. Mirabegron add-on therapy to tamsulosin for the treatment of overactive bladder in men with lower urinary tract symptoms: a randomized, placebo-controlled study (MATCH). Eur Urol Focus. 2020;6(4):729-37.

33. Benner JS, Nichol MB, Rovner ES, Jumadilova Z, Alvir J, Hussein M, Fanning K, Trocio JN, Brubaker L. Patient-reported reasons for discontinuing overactive bladder medication. BJU Int. 2010;105(9):1276-82.

34. Yeowell G, Smith P, Nazir J, Hakimi Z, Siddiqui E, Fatoye F. Real-world persistence and adherence to oral antimuscarinics and mirabegron in patients with overactive bladder $(\mathrm{OAB})$ : a systematic literature review. BMJ Open. 2018;8(11):e021889. 
35. Drake MJ, Bowditch S, Arbe E, Hakimi Z, Guelfucci F, Amri I, Nazir J. A retrospective study of treatment persistence and adherence to alpha-blocker plus antimuscarinic combination therapies, in men with LUTS/BPH in the Netherlands. BMC Urol. 2017;17(1):36.

36. Eisen C, Lulic Z, Palacios-Moreno JM, Adalig B, Hennig M, Cortes V, Gilg F, Kostev K. Persistence and adherence to dutasteride/tamsulosin fixeddose versus free-combination alpha blocker/5ARI therapy in patients with benign prostate hyperplasia in Germany. Int J Clin Pharmacol Ther. 2020;58(1):37-49

\section{Publisher's Note}

Springer Nature remains neutral with regard to jurisdictional claims in published maps and institutional affiliations.
Ready to submit your research? Choose BMC and benefit from:

- fast, convenient online submission

- thorough peer review by experienced researchers in your field

- rapid publication on acceptance

- support for research data, including large and complex data types

- gold Open Access which fosters wider collaboration and increased citations

- maximum visibility for your research: over $100 \mathrm{M}$ website views per year

At BMC, research is always in progress.

Learn more biomedcentral.com/submissions 